\title{
Smooth muscle cell-specific fibronectin-EDA mediates phenotypic switching and neointimal hyperplasia
}

\author{
Manish Jain, ${ }^{1}$ Nirav Dhanesha, ${ }^{1}$ Prakash Doddapattar, ${ }^{1}$ Mehul R. Chorawala, ${ }^{1}$ Manasa K. Nayak, ${ }^{1}$ Anne Cornelissen, ${ }^{2}$ Liang Guo, ${ }^{2}$ \\ Aloke V. Finn, ${ }^{2}$ Steven R. Lentz, ${ }^{1}$ and Anil K. Chauhan ${ }^{1}$ \\ 'Department of Internal Medicine, Division of Hematology/Oncology, University of lowa, lowa City, lowa, USA. ²CPath Institute Inc., Gaithersburg, Maryland, USA.
}

\begin{abstract}
Fibronectin-splice variant containing extra domain A (Fn-EDA) is associated with smooth muscle cells (SMCs) following vascular injury. The role of SMC-derived Fn-EDA in SMC phenotypic switching or its implication in neointimal hyperplasia remains unclear. Herein, using human coronary artery sections with a bare metal stent, we demonstrate the expression of Fn-EDA in the vicinity of SMC-rich neointima and peri-strut areas. In mice, Fn-EDA colocalizes with SMCs in the neointima of injured carotid arteries and promotes neointima formation in the comorbid condition of hyperlipidemia by potentiating SMC proliferation and migration. No sex-based differences were observed. Mechanistic studies suggested that Fn-EDA mediates integrin- and TLR4-dependent proliferation and migration through activation of FAK/Src and Akt1/mTOR signaling, respectively. Specific deletion of Fn-EDA in SMCs, but not in endothelial cells, reduced intimal hyperplasia and suppressed the SMC synthetic phenotype concomitant with decreased Akt1/mTOR signaling. Targeting Fn-EDA in human aortic SMCs suppressed the synthetic phenotype and downregulated Akt1/mTOR signaling. These results reveal that SMC-derived Fn-EDA potentiates phenotypic switching in human and mouse aortic SMCs and neointimal hyperplasia in the mouse. We suggest that targeting Fn-EDA could be explored as a potential therapeutic strategy to reduce neointimal hyperplasia.
\end{abstract}

\section{Introduction}

Percutaneous coronary intervention (PCI) with stent implantation is commonly performed for the treatment of coronary artery disease. Neointimal hyperplasia with smooth muscle cell (SMC) migration and proliferation and extracellular matrix (ECM) deposition and remodeling remains a significant factor limiting the success of PCI $(1,2)$. In healthy arteries, SMCs are surrounded by a basement membrane composed of laminin, collagen type IV, and heparan sulfate proteoglycan. In contrast, SMC activation following arterial injury is associated with the disappearance of laminin and other basement membrane structures and the appearance of abundant deposits of fibronectin (Fn) around proliferative cells in the media and intima $(3,4)$, suggesting a functional role for Fn in neointimal formation.

Fn plays an essential role in several cellular processes as well as hemostasis and thrombosis $(5,6)$. Fn exhibits diversity at the protein level as a consequence of alternative splicing of a single primary transcript at 3 exons that encode extra domain A (EDA), extra domain B (EDB), and the type III homologies connecting segment. Fn found in plasma (pFn) lacks both the EDA and EDB segments and is synthesized by hepatocytes. The predominant isoforms of Fn found in the ECM, which is known as cellular Fn $(\mathrm{cFn})$, are dimeric or cross-linked multimeric structures contain-

Conflict of interest: The authors have declared that no conflict of interest exists. Copyright: $(2020$, American Society for Clinical Investigation. Submitted: November 19, 2018; Accepted: September 25, 2019;

Published: November 25, 2019

Reference information: J Clin Invest. 2020;130(1):295-314.

https://doi.org/10.1172/JCl124708. ing either EDA or EDB or both, in varying proportions. cFn containing EDA (Fn-EDA) in the ECM is synthesized by vascular cells, including endothelial cells and SMCs (5). The EDA amino acid sequence is highly conserved (>90\%) in mammals, including mice, rats, and humans (7). Fn-EDA is implicated in wound healing $(8,9)$, vascular intimal proliferation $(10)$, and fibrotic disorders of the lung, liver, and skin $(11,12)$. pFn and $\mathrm{cFn}$ bind to several integrins, including $\alpha_{5} \beta_{1}, \alpha_{3} \beta_{1}, \alpha_{\mathrm{v}} \beta_{1}$, and $\alpha_{\mathrm{v}} \beta_{3}$, that are expressed on SMCs. Although $\mathrm{pFn}$ can promote modulation of SMC from contractile to synthetic phenotype via an integrin-dependent mechanism (4), Fn-EDA is a characteristic feature of only synthetic SMCs (10). In addition to integrins, Fn-EDA is a ligand for the innate immune receptor Toll-like receptor 4 (TLR4). Fn-EDA activation of TLR4 requires the myeloid differentiation-2 (MD-2) receptor (13). Both TLR4 and integrin signaling pathways are known to be involved in SMC proliferation and neointimal hyperplasia $(14,15)$. Fn-EDA may, therefore, modulate SMC function via potentiation of both TLR4 and integrin signaling pathways. We and others have shown that genetic deletion of Fn-EDA in murine models reduces atherosclerosis and improves stroke outcome by limiting thrombosis and inflammation (16-20). The specific role of Fn-EDA in the pathology of neointimal hyperplasia has not been elucidated yet.

Herein, we determined the mechanistic role of Fn-EDA in neointimal hyperplasia in both normolipidemic and hyperlipidemic conditions. We chose hyperlipidemia in addition to normolipidemia for the following reasons: (a) Patients with hyperlipidemia have a higher risk of developing restenosis in the vascular bed (21, 22), and human PCI procedures are often performed in patients with comorbid conditions such as hyperlipidemia. (b) Although experiments done in wild-type (WT) mice are very useful in dis- 


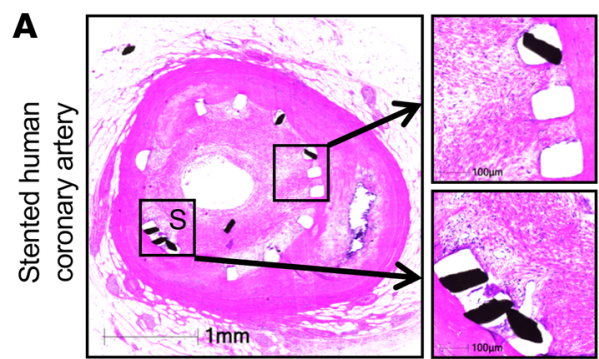

Hematoxylin and Eosin

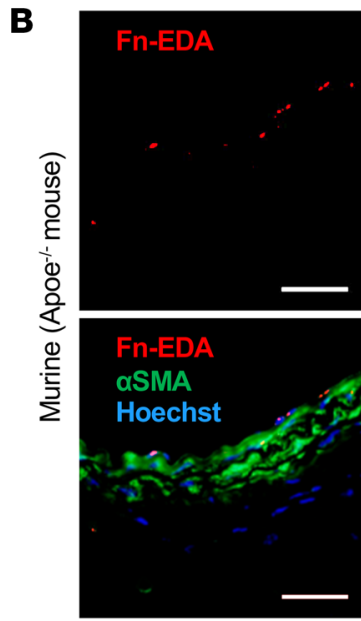

Uninjured

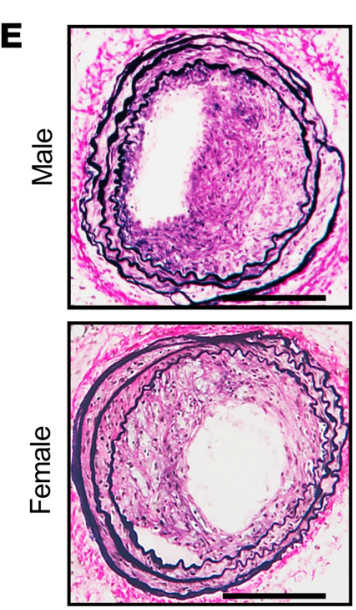

$\mathrm{Apoe}^{-/ 2}$

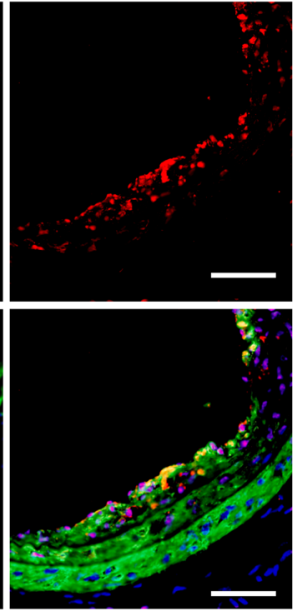

Day 14

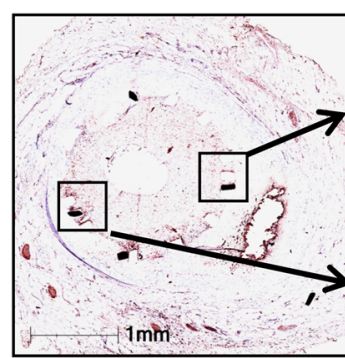

Fn-EDA
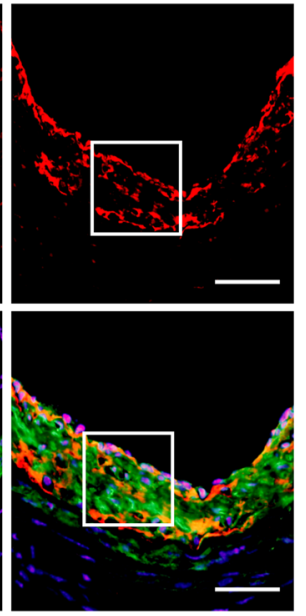

Day 28

Injured carotid
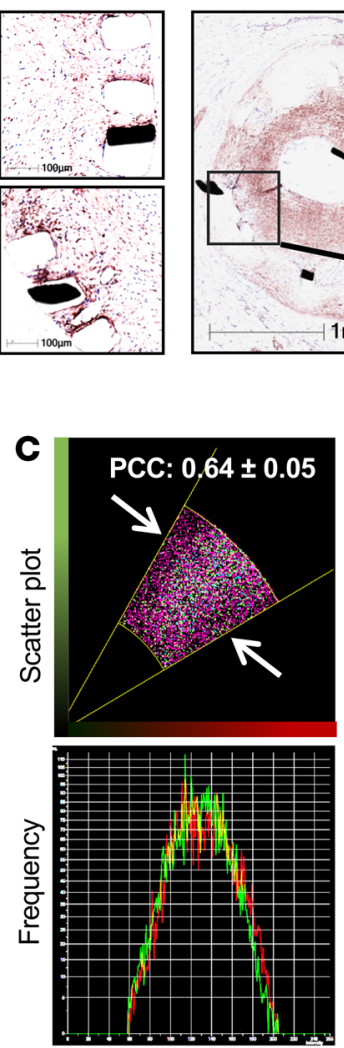

Intensity

colocalization

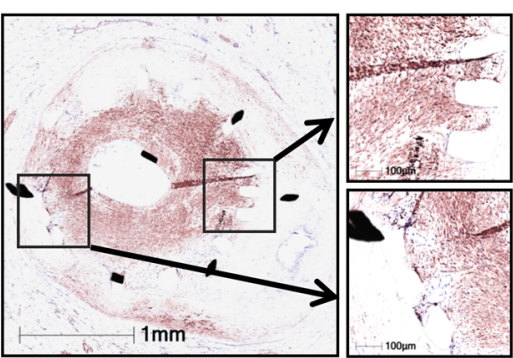

aSMA
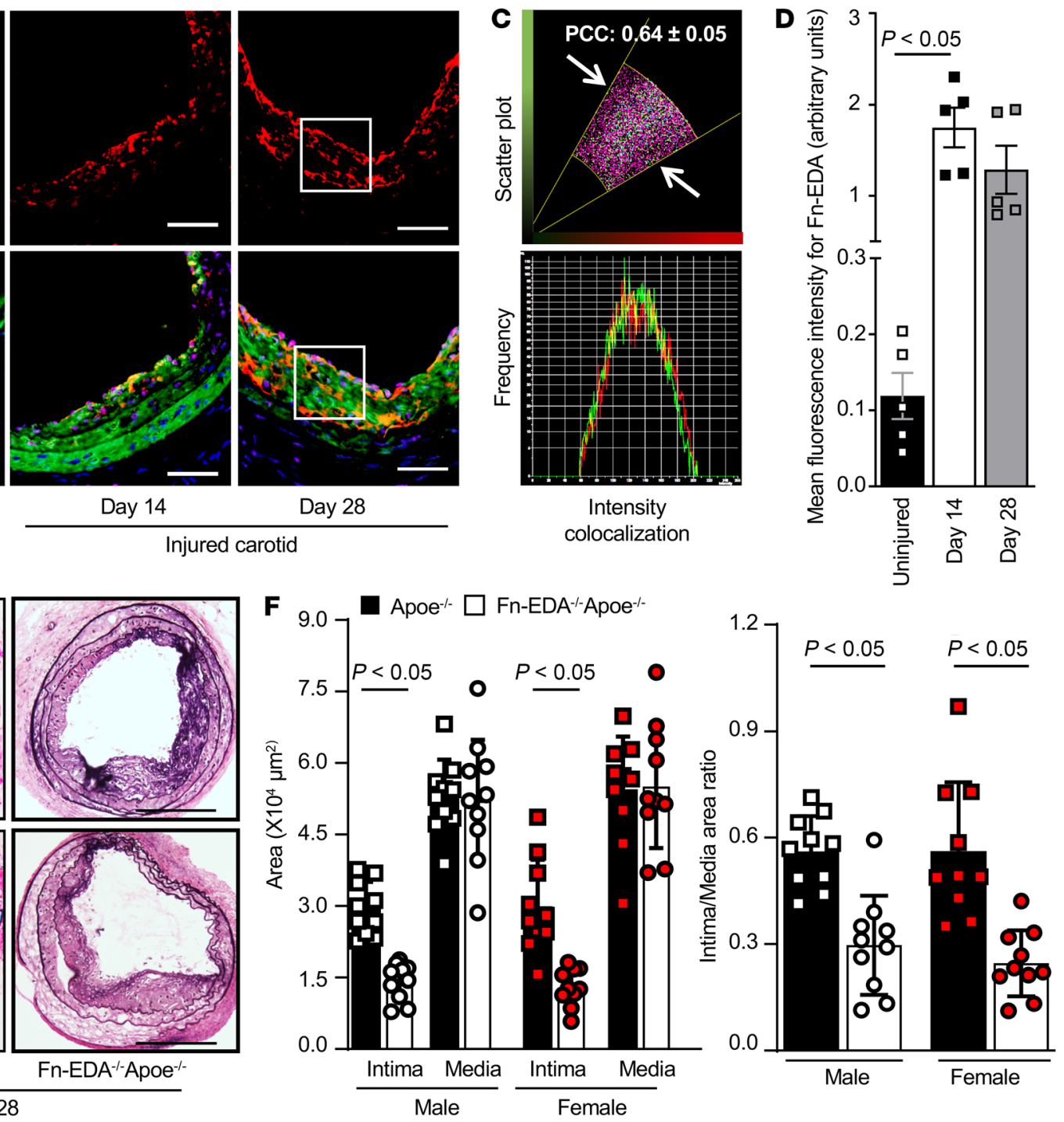

Figure 1. Detrimental role of Fn-EDA in intimal hyperplasia. (A) Representative images of cross-sectional immunohistochemistry in stented human coronary arteries showed Fn-EDA in SMC-rich neointima and peri-strut areas. Left panel shows H\&E staining, middle panel shows Fn-EDA, and right panel shows $\alpha$-smooth muscle actin ( $\alpha S M A)$ staining. Boxed regions are magnified. " $S$ " denotes strut in the cross sections; dark areas are pieces of strut sections. (B) Representative images showing double immunostaining for Fn-EDA (red) and SMCs (green) in the uninjured and injured carotid artery of Apoe $^{-/-}$mice harvested after 14 and 28 days of injury ( $n=5-6$ per group). Nuclei are counterstained with Hoechst (blue). Boxed regions are magnified. Scale bars: $50 \mu \mathrm{m}$. (C) Representative scatter plot and intensity profile demonstrating colocalized pixels and pixel intensity for both channels (Fn-EDA, red, $x$ axis; and SMC, green, $y$ axis) with Pearson's correlation coefficient (PCC). Colocalized pixels are defined as those whose intensity values for both channels fall within a preset range above the background intensity level (white arrows). (D) Quantification of the Fn-EDA fluorescence intensity. (E) Representative photomicrographs of Verhoeff's/van Gieson-stained carotid artery sections of male and female Fn-EDA ${ }^{-{ }^{-}}$Apoe $^{-/-}$and Apoe ${ }^{-/-}$mice after 28 days of injury ( $n=10$ per group). Scale bars: $200 \mu \mathrm{m}$. (F) Quantification shows intimal area, medial area, and a ratio of intimal to medial area. Each dot represents a single mouse. Values are represented as mean \pm SEM. Statistical analysis: unpaired Student's $t$ test. 
secting mechanisms of injury-induced intimal hyperplasia, inbred strains of WT mice do not model the effects of clinically relevant comorbid conditions such as hyperlipidemia. Using mouse aortic isolated SMCs, several mutant mouse models, and human aortic SMC cultures, we demonstrate that SMC-derived Fn-EDA mediates SMC phenotypic switching and plays a mechanistic role in neointimal hyperplasia.

\section{Results}

Fn-EDA is expressed in stented human coronary artery sections and mediates neointimal hyperplasia in mice. We first determined the expression of Fn-EDA in stented human coronary arteries. We focused on samples in which bare metal stents had been implanted for 60-120 days (mean duration of implant 86.1 \pm 24.7 days) having active progression of neointima and SMC proliferation (23). We identified 6 such samples that had the same type of stent from the CVPath Institute Stent Registry. Patient coronary artery disease status and stent characteristics are shown in Supplemental Table 1 (supplemental material available online with this article; https://doi.org/10.1172/JCI124708DS1). Immunohistochemistry (IHC) demonstrated the expression of Fn-EDA in the vicinity of SMC-rich neointima and peri-strut areas (Figure 1A). Next, we determined the role of the Fn-EDA in neointima formation under normolipidemic and hyperlipidemic (a causative factor of coronary artery disease) conditions. Susceptibility to neointimal hyperplasia was studied at 28 days following wire injury in the carotid artery. We found marked Fn-EDA expression in the neointima at 28 days after wire injury, which colocalized with $\alpha$-smooth muscle actin $(\alpha \mathrm{SMA})$ staining $(P<0.05$ vs. uninjured artery, Pearson's correlation 0.61; Supplemental Figure 1, A-C). Genetic deletion of Fn-EDA in WT mice resulted in a significant decrease in the neointimal area as well as the intimal/medial area ratio $(P<$ 0.05 vs. WT mice; Supplemental Figure 1, D and E). No Fn-EDA signal was observed in the negative controls incubated with isotype-matched Igs (Supplemental Figure 2A). Similarly, Fn-EDA staining was virtually absent in injured carotid arteries or isolated SMCs of Fn-EDA ${ }^{-/}$mice (Supplemental Figure 2B).

To assess the role of Fn-EDA in neointima formation in the comorbid condition of hyperlipidemia (an independent risk factor associated with restenosis) (24), we used Fn-EDA ${ }^{-/-}$mice on the hyperlipidemic apolipoprotein E-deficient background

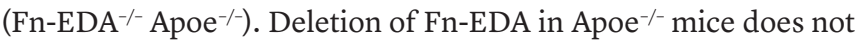
affect plasma lipid levels or complete blood counts (16-18). In line with WT studies, we found that the majority of Fn-EDA colocalized with $\alpha$ SMA staining in the neointima of carotid arteries of Apoe $^{-/-}$mice at 14 and 28 days following wire injury $(P<0.05$ vs. uninjured artery, Pearson's correlation 0.64; Figure 1, B-D). Additionally, Fn-EDA staining colocalized with endothelial cells (CD31) (13.5\% $\pm 1.2 \%)$ and macrophages (CD68) (4.8\% $\pm 0.4 \%)$; however, colocalization was markedly less in these cells than in SMCs ( $\alpha \mathrm{SMA})(40 \% \pm 3.4 \%$; Supplemental Figure 3$)$. Next, we determined susceptibility to neointimal hyperplasia at 28 days following wire injury. Male and female mice were examined separately to determine sex-based differences. To minimize the potential confounding effect of advanced atherosclerotic lesions, which can impair flow and indirectly exacerbate the effect of wire injury, Fn-EDA ${ }^{-/-} \mathrm{Apoe}^{-/-}$and control Apoe ${ }^{-/-}$mice were fed a standard chow diet until 10-12 weeks of age, an age at which no hemodynamically significant vascular lesions are found (not shown). Both male and female Fn-EDA ${ }^{-/-} \mathrm{Apoe}^{-/-}$mice exhibited marked ( $\left.50 \%\right)$ decreases in the neointimal area as well as neointimal/medial ratio $\left(P<0.05\right.$ vs. Apoe ${ }^{-/}$mice, $n=9-10$ mice per group; Figure 1 , $\mathrm{E}$ and $\mathrm{F})$. No sex-based differences were observed. Body weights were comparable (not shown).

$\mathrm{cFn}$ is composed of dimeric or cross-linked multimeric structures containing EDA and/or EDB in varying proportions. It is possible that the deletion of Fn-EDA may affect the total amount of Fn or Fn-EDB expression or the quality of Fn fibers that may indirectly reduce neointimal hyperplasia. To check this possibility, we first quantified expression levels of Fn-EDB and total Fn in SMCs isolated from Apoe ${ }^{-/-}$and Fn-EDA ${ }^{-/-} \mathrm{Apoe}^{-/-}$mice. Fn-EDB levels were comparable as analyzed by quantitative reverse transcriptase PCR (RT-PCR) (Supplemental Figure 4). These results are in agreement with previous studies suggesting the autonomy of the Fn-EDB alternatively spliced form in adult mice lacking Fn-EDA (25). Total Fn protein levels were comparable in SMCs and in the neointima of injured carotid arteries of $\mathrm{Apoe}^{-/-}$and Fn-EDA ${ }^{-/-}$ Apoe $^{-/-}$mice as analyzed by Western blot and IHC (Supplemental Figure 5, A-C). Next, we assessed the quality of Fn fibers by IHC. No morphological differences were noted in terms of thickness or length of Fn fibers between SMCs from Apoe A- $^{-/}$and Fn-EDA ${ }^{-/-}$ Apoe $^{-/-}$mice (Supplemental Figure 5D). SMCs from both groups assembled fibrillar Fn at the basal cell surface and formed a prominent network of thick Fn fibers that were aligned in parallel to actin fibers. Together, these results suggest that reduced neointimal hyperplasia observed in Fn-EDA-deficient mice is most likely independent of Fn-EDB isoform or quality or quantity of Fn fibers.

Fn-EDA deletion suppresses SMC proliferation and migration. Previously, it was shown that on a pFn-coated surface and cultured in a defined, serum-free medium, arterial SMCs rapidly and efficiently transform into a synthetic phenotype (26). On the other hand, evidence suggests that serum-starved SMCs undergo complete redifferentiation, during which they become elongated and spindle-shaped with elevation of SMC-specific contractile proteins including SM22 $\alpha$ and SM-MHC $(27,28)$. Therefore, we stimulated aortic SMCs with platelet-derived growth factor-BB (PDGF). Immunostaining and quantitative PCR (qPCR) revealed that aortic SMCs stimulated with PDGF expressed Fn-EDA (Figure 2, A and B), suggesting that Fn-EDA is a PDGF-induced gene. Next, we determined whether Fn-EDA promotes SMC proliferation and migration. PDGF-BB-stimulated aortic SMCs from Apoe $^{-/-}$mice and Fn-EDA ${ }^{-/-}$Apoe $^{-/-}$mice were subjected to BrdU incorporation assays. SMCs lacking Fn-EDA proliferated less when compared with SMCs containing Fn-EDA (16.9\% $\pm 1.5 \%$ vs. $37.8 \% \pm 1.1 \%$; Figure $2 \mathrm{C}$ ). Consistent with this finding, PDGF-BBstimulated aortic SMCs from Apoe ${ }^{-/-}$mice exhibited an increase in the percentage of cells in synthetic (S) phase in comparison with Fn-EDA ${ }^{-/-}$Apoe $^{-/-}$mice $(24.9 \% \pm 2.2 \%$ vs. $16.3 \% \pm 1.7 \%$; Figure 2D and Supplemental Figure 6). Next, we examined the role of Fn-EDA in SMC migration in response to PDGF-BB. Aortic SMCs were grown to $90 \%$ confluence, and in vitro scratch assays were performed. We found that Fn-EDA deletion reduced PDGF-BBinduced SMC migration (Figure 2E). Together, these results suggest that Fn-EDA potentiates SMC proliferation and migration. 


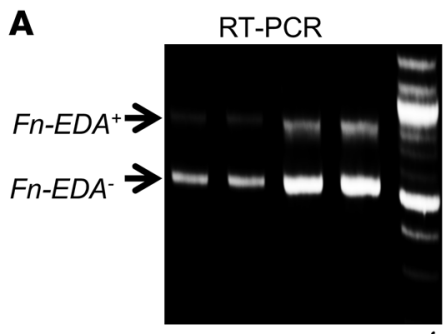

Time (hours) $\begin{array}{llll}0 & 6 & 12 & 24\end{array}$

PDGF-BB-stimulated aortic SMC culture from $A_{p o e^{-/-}}$mouse
B

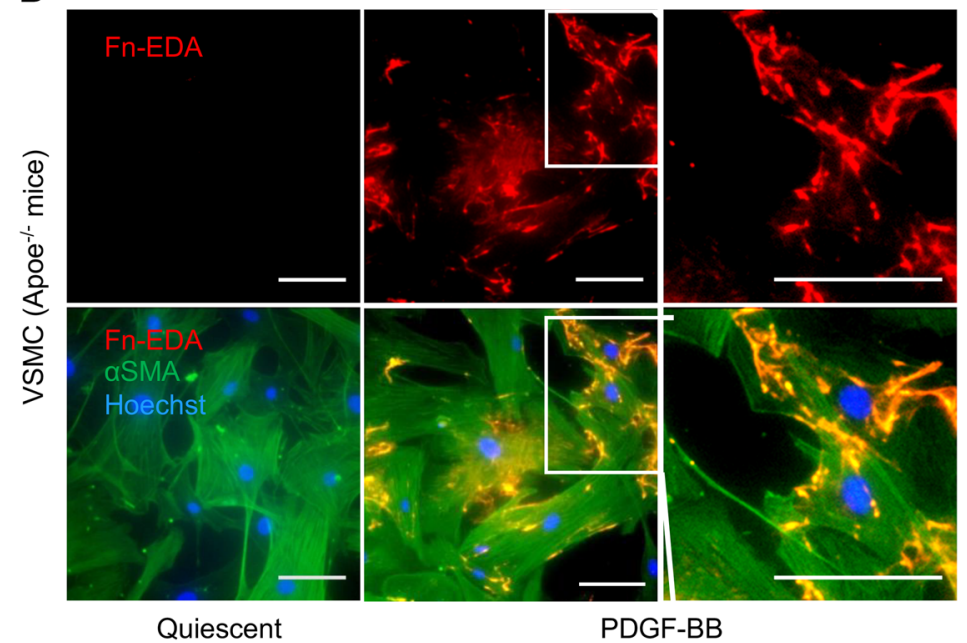

PDGF-BB

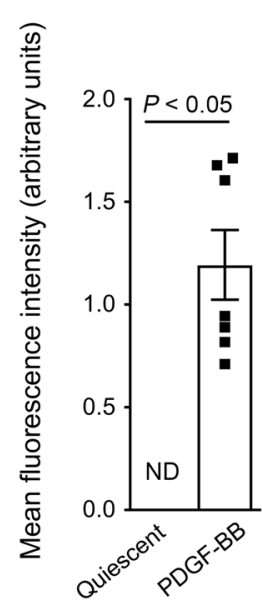

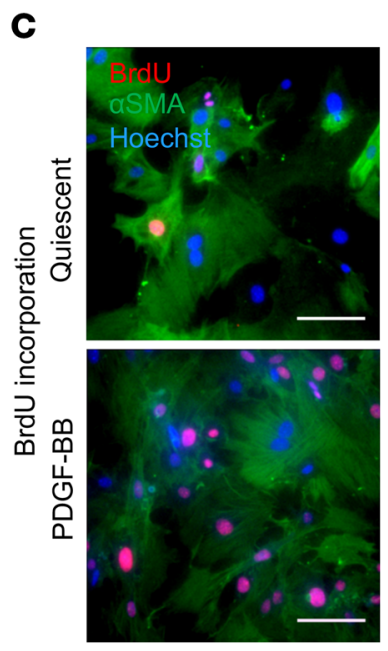

$\mathrm{Apoe}^{-/-}$

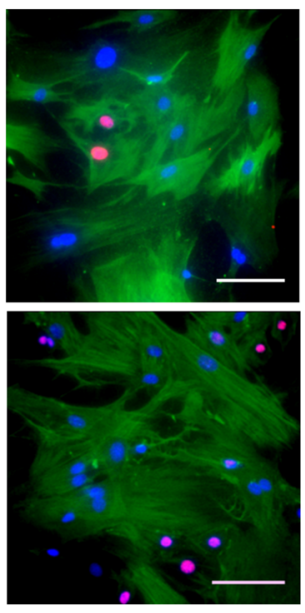

Fn-EDA ${ }^{-1-}$ Apoe $^{-/-}$

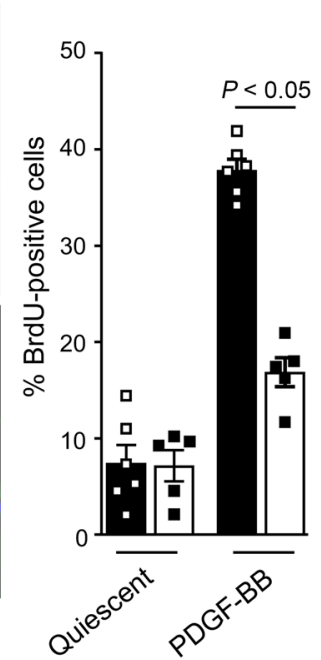

E

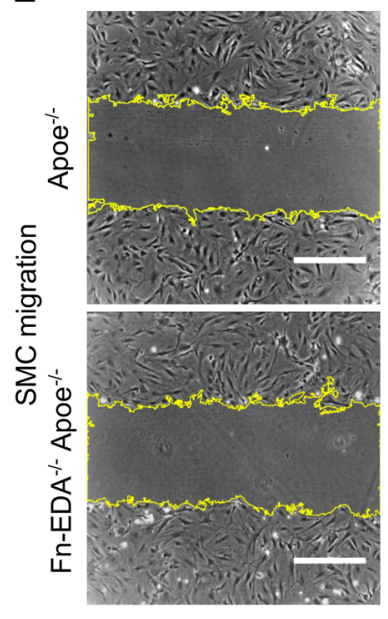

Time

$0 \mathrm{hrs}$

PDGF-BB

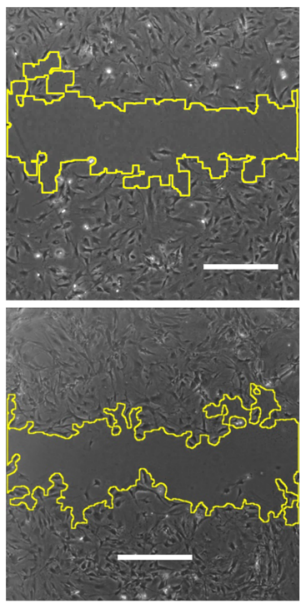

$24 \mathrm{hrs}$

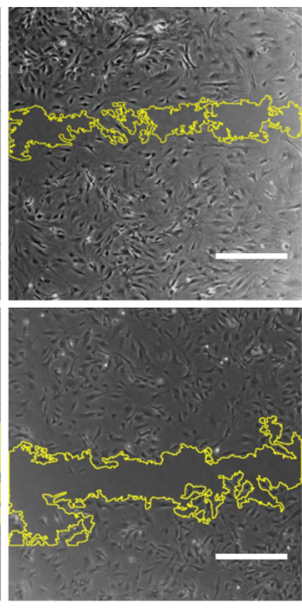

$24 \mathrm{hrs}$
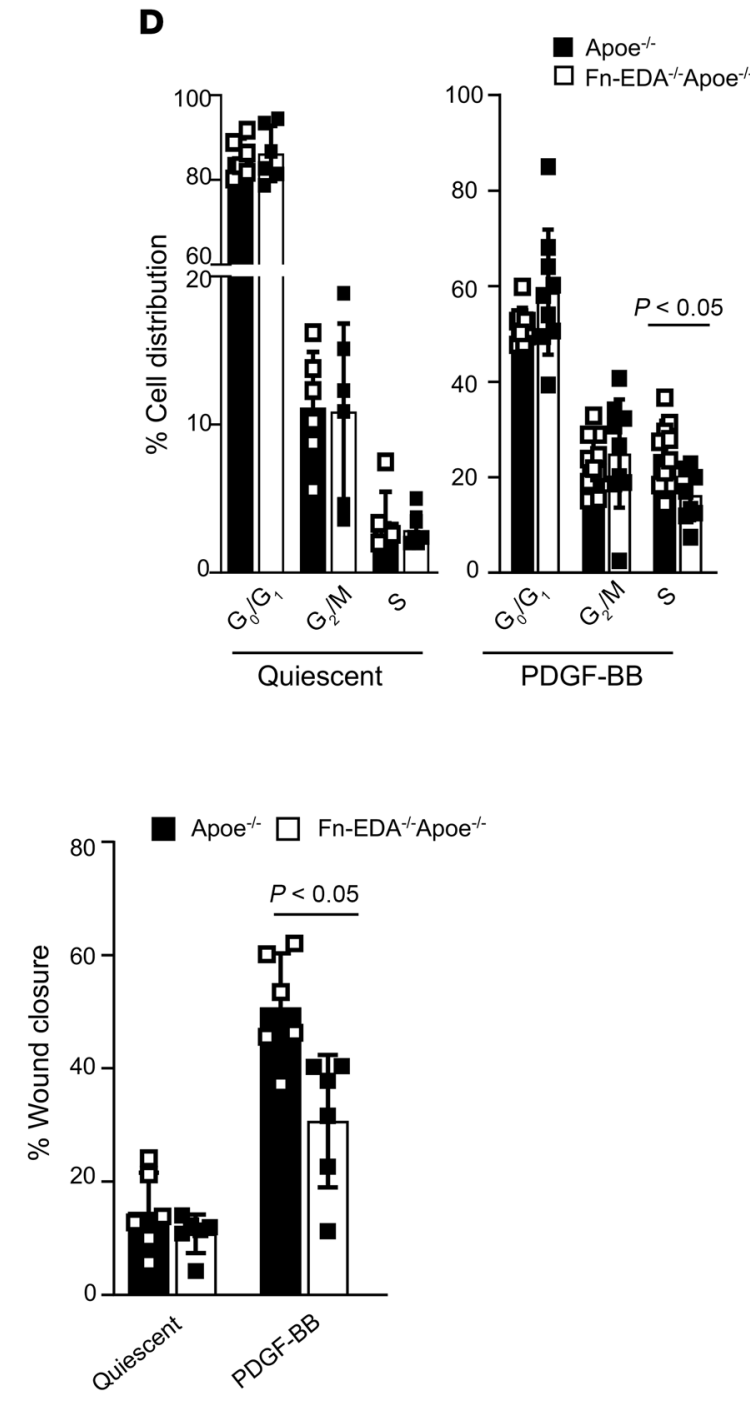
Figure 2. Fn-EDA deletion in Apoe $^{-/-}$mice suppresses SMC proliferation and migration. Aortic SMCs isolated from Apoe ${ }^{-/-}$and Fn-EDA ${ }^{-1-} \mathrm{Apoe}^{-1-}$ mice were serum-starved for 48 hours and stimulated with or without PDGF-BB for 24 hours. (A) RT-PCR showing the mRNA expression of Fn-EDA at different time points in PDCF-BB-stimulated SMCs. (B) The left panels show representative double immunostaining for Fn-EDA (red) and SMC (green). The right panel shows the quantification of Fn-EDA ( $n$ $=6$ per group). Scale bars: $25 \mu \mathrm{m}$. ND, not detected. (C) The left panels show representative BrdU-positive cells costained with $\alpha \mathrm{SMA}$ (green) and Hoechst (blue). Scale bars: $50 \mu \mathrm{m}$. The right panel shows the quantification of BrdU-positive cells to the total number of cells ( $n=6$ per group). (D) Quiescent or PDCF-BB-stimulated aortic SMCs were stained with propidium iodide, and cell cycle was analyzed using flow cytometry. The panels show quantitative data of cell cycle distribution in quiescent and PDGF-BB-stimulated SMCs ( $n=6-8$ per group). (E) The left panels show representative phase-contrast images of SMC migration in the scratch assay. The right panel shows quantification of the migrated area $(n=6-8$ per group). Scale bars: $500 \mu \mathrm{m}$. Values are expressed as mean \pm SEM. Statistical analysis: unpaired Student's $t$ test.

Fn-EDA mediates phenotypic switching by potentiating Akt1/ mTOR signaling. SMC modulation from a differentiated "contractile" to a dedifferentiated "synthetic" proliferative phenotype contributes to SMC proliferation, migration, and thereby intimal hyperplasia. To elucidate the mechanistic role of Fn-EDA on SMC phenotypic switching, aortic SMCs were immunostained for contractile and synthetic phenotype markers. We found that the expression levels of contractile markers (SM22 $\alpha$ and SM-MHC) were increased whereas synthetic markers (vimentin and osteopontin) were decreased in SMCs isolated from $\mathrm{Fn}-\mathrm{EDA}^{-/-} \mathrm{Apoe}^{-/-}$ mice $\left(P<0.05\right.$ vs. Apoe ${ }^{-/-}$mice; Figure $\left.3 \mathrm{~A}\right)$. The results were confirmed in parallel in SMC lysates by Western blot (Figure 3B). Next, we investigated whether Fn-EDA potentiates synthetic phenotype via the Akt1/mTOR signaling pathway, which is known to regulate the cell cycle, proliferation, and migration (29). We found that PDGF-BB-stimulated aortic SMCs from Fn-EDA ${ }^{-/-} \mathrm{Apoe}^{-/-}$mice exhibited reduced phospho-Akt1 (0.67-fold), phospho-mTOR (0.55-fold), and phospho-NF-кB p65 (0.47-fold) in comparison with Apoe $^{-/-}$mice (Figure 3C). In contrast, Akt2, which is known to exert antiproliferative and antimigratory effects (30), was increased in aortic SMCs from Fn-EDA ${ }^{-/} \mathrm{Apoe}^{-/-}$mice when compared with Apoe ${ }^{-/-}$mice (Figure 3C). Furthermore, we found that absence of Fn-EDA reduced TNF- $\alpha$ and IL-1 $\beta$ secretion from aortic SMCs $\left(P<0.05\right.$ vs. Apoe ${ }^{-/}$mice; Figure 3D). Mechanistically, these results suggest that Fn-EDA promotes phenotypic switching by upregulating Akt1/mTOR signaling.

Exogenous Fn-EDA potentiates phenotypic switching in aortic SMCs. We next investigated whether exogenous cFn (which contains EDA) can potentiate the Akt1/mTOR signaling pathway in a dose-dependent manner and mediate SMC phenotypic switching. Aortic SMCs from Fn-EDA ${ }^{-/-}$Apoe $^{-/-}$mice were treated for 24 hours with human $\mathrm{cFn}(0-50 \mu \mathrm{g} / \mathrm{mL})$ in serum-free medium. We then measured levels of phospho-Akt1, phospho-mTOR, phospho-NF- $\mathrm{B}$ p65, and phospho-Akt2 in lysates and the inflammatory cytokines TNF- $\alpha$ and IL-1 $\beta$ in the medium. We found that, at a threshold dose of $20 \mu \mathrm{g} / \mathrm{mL}$, cFn potentiated Akt1/mTOR signaling associated with an increase in TNF- $\alpha$ and IL- $1 \beta$ protein levels (Figure 4, A and B). We next determined whether $\mathrm{cFn}$ at a threshold dose of $20 \mu \mathrm{g} / \mathrm{mL}$ could potentiate SMC phenotypic switching in the absence of PDGF-BB. Aortic SMCs from Fn-EDA ${ }^{-/-} \mathrm{Apoe}^{-/-}$ mice were treated with $20 \mu \mathrm{g} / \mathrm{mL}$ of cFn for 24 hours and immunostained for contractile (SM22 $\alpha$ and SM-MHC) and synthetic (vimentin and osteopontin) phenotype markers. Control aortic SMCs from Fn-EDA ${ }^{-/}$Apoe Ap $^{-/}$mice were treated with $20 \mu \mathrm{g} / \mathrm{mL}$ of $\mathrm{pFn}$ (which does not contain EDA). We found that expression levels of SM22 $\alpha$ and SM-MHC were reduced in cFn-treated SMCs $(P<$ 0.05 vs. pFn-treated SMCs; Figure 4C). In contrast, the expression levels of vimentin and osteopontin were increased in $\mathrm{cFn}$-treated SMCs $(P<0.05$ vs. pFn-treated SMCs; Figure $4 C)$. These findings were confirmed in parallel in aortic SMC lysates by Western blot (Figure 4D). Together, these results suggest that exogenous cFn potentiates Akt1/mTOR signaling and phenotypic switching in aortic SMCs. cFn contains both Fn-EDA and Fn-EDB isoforms. To confirm the specific role of Fn-EDA in potentiating phenotypic switching in aortic SMCs, we treated SMCs from Fn-EDA ${ }^{-/} \mathrm{Apoe}^{-/-}$ mice with recombinant EDA-containing or EDA-lacking peptides $(10 \mu \mathrm{g} / \mathrm{mL})$. We found that recombinant EDA-containing peptide significantly increased the expression levels of synthetic markers (vimentin and osteopontin) and decreased the expression of contractile markers (SM22 $\alpha$ and SM-MHC) (Supplemental Figure 7A). Concomitantly, treatment of SMCs from Fn-EDA ${ }^{-/-}$Apoe $^{-/-}$mice with EDA-containing peptides led to a significant increase in SMC proliferation and migration (Supplemental Figure 7B). Although these results do not exclude a role for EDB, they suggest that most likely Fn-EDA is a primary cFn component that potentiates phenotypic switching.

Fn-EDA promotes proliferation and migration in aortic SMCs through both integrin- and TLR4-dependent signaling. Previously, using a synthetic RGDS peptide (Arg-Gly-Asp-Ser), it was shown that $\mathrm{pFn}$ induces phenotypic modulation and proliferation of isolated rat arterial SMCs, suggesting a role for $\mathrm{pFn}$ /integrin signaling in SMC phenotypic switching (26). First, we determined whether integrin signaling was disrupted in SMCs isolated from Fn-EDA ${ }^{-/} \mathrm{Apoe}^{-/-}$mice. We found that PDGF-BB-stimulated aortic SMCs from $\mathrm{Fn}_{-\mathrm{EDA}^{-/}} \mathrm{Apoe}^{-/-}$mice exhibited reduced phospho-FAK ( 0.6 fold) and phospho-SRC ( 0.5 fold) when compared with Apoe $^{-/-}$mice $(P<0.05$; Supplemental Figure 8$)$, suggesting defective integrin signaling in Fn-EDA ${ }^{-/-} \mathrm{Apoe}^{-/-}$mice. Next, we determined whether Fn-EDA-mediated SMC phenotypic switching and proliferation is integrin-dependent. SMCs from Fn-EDA ${ }^{-/-}$ Apoe $^{-/-}$mice were pretreated with RGDS peptide and stimulated with cFn (contains EDA) or pFn (lacks EDA). We found that RGDS peptide inhibited both $\mathrm{pFn}$ - and $\mathrm{cFn}$-mediated SMC phenotypic switching and proliferation; however, the extent of proliferation and migration was still significantly higher in SMCs stimulated with RGDS peptide plus cFn versus RGDS peptide plus pFn (Figure 5). This observation suggested the involvement of other endogenous Fn-EDA receptors, such as TLR4, which are not recognized by the RGDS peptide.

Next, we determined the role of TLR4 in Fn-EDA-mediated SMC phenotypic switching and proliferation. Immunoprecipitation studies with PDGF-BB-stimulated aortic SMC lysates from Apoe $^{-/-}$mice confirmed that Fn-EDA interacts with TLR4 on SMCs (Figure 6A). Next, aortic SMCs from Fn-EDA ${ }^{-/-} \mathrm{Apoe}^{-/-}$and Fn-EDA ${ }^{-/-}$TLR4 $^{-/-}$Apoe $^{-/-}$mice were stimulated for 24 hours in the presence of $\mathrm{pFn}$ or $\mathrm{cFn}$. We found that exogenous $\mathrm{cFn}$ pro- 
A
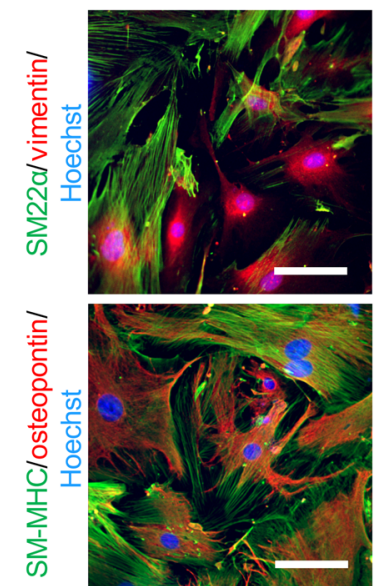

Apoe $^{-1}$

B
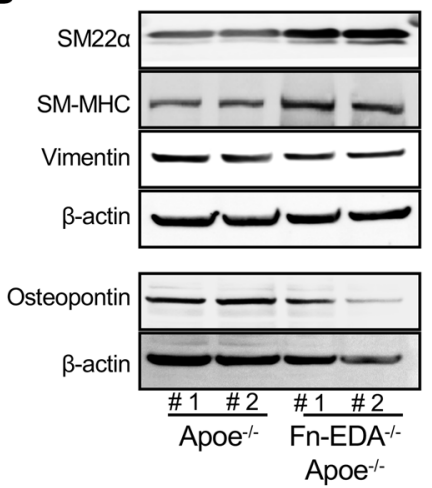

C

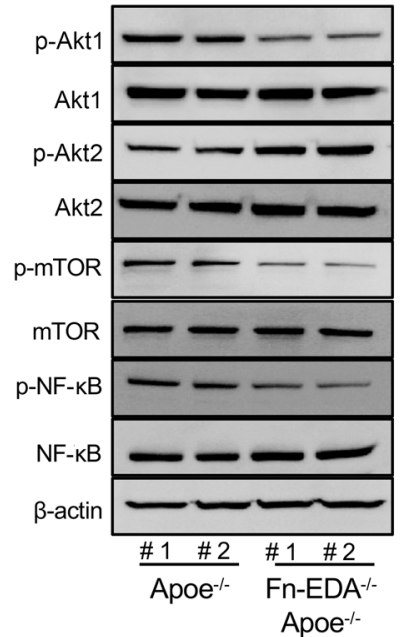

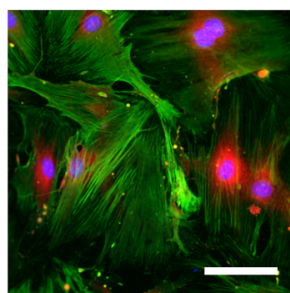

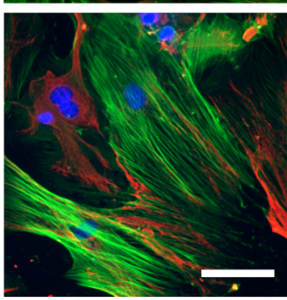

Fn-EDA ${ }^{-/}$Apoe $^{-/-}$
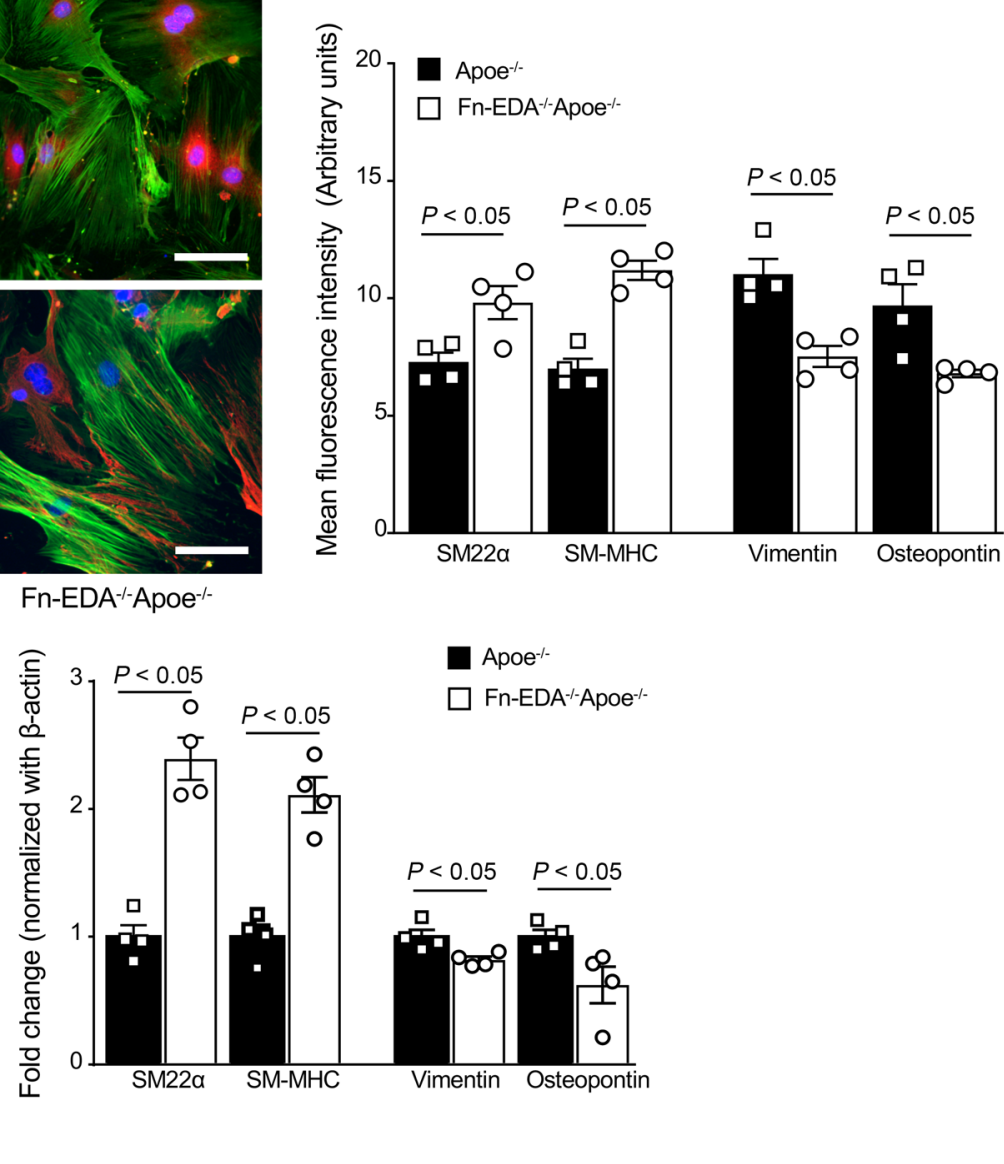

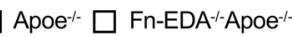

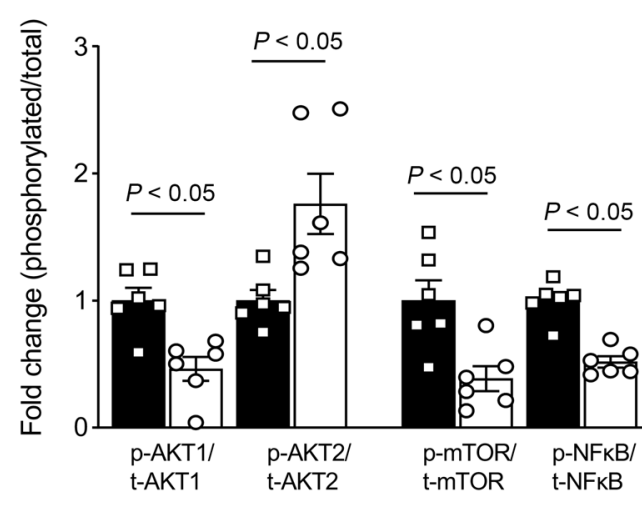

D

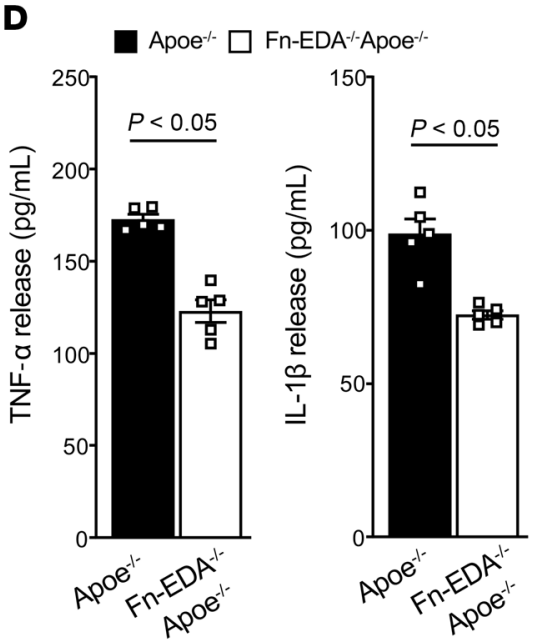

Figure 3. Fn-EDA deletion in Apoe ${ }^{-/-}$mice suppresses synthetic phenotype and attenuates Akt1 signaling. Aortic SMCs isolated from Apoe ${ }^{-/-}$and Fn-EDA ${ }^{-/-} \mathrm{Apoe}^{-/-}$mice were serum-starved for 48 hours and stimulated with or without PDGF-BB for 24 hours. (A) The left panels show representative immunostaining images for contractile proteins (SM22 $\alpha$, green; and SM-MHC, green) and synthetic proteins (vimentin, red; and osteopontin, red). Scale bars: $25 \mu \mathrm{m}$. The right panel shows quantification of the fluorescence intensity for SM22 $\alpha$, SM-MHC, vimentin, and osteopontin ( $n=4$ per group). (B) Representative immunoblots and densitometric analysis of SM22 $\alpha$, SM-MHC, vimentin, and osteopontin ( $n=4$ per group). Blots for SM22 $\alpha$, SM-MHC, and vimentin are from the same biological samples. (C) Quiescent aortic SMCs were stimulated with PDCF-BB (20 ng/mL) for $30 \mathrm{minutes}$, and cells were processed for Western blotting. Duplicate samples were run in the same gels, with the membrane cut in half, and then probed for total and phosphorylated proteins separately. Representative Western blots and densitometric analysis of Akt1, Akt2, mTOR, NF-kB, and $\beta$-actin ( $n=6$ per group). \# 1 and \# 2 are samples from 2 different experiments. (D) Serum-starved aortic SMCs were stimulated with PDGF-BB for 24 hours, and cell supernatant was used for quantification of TNF- $\alpha$ and IL-1 $\beta$ by ELISA ( $n=5$ per group). Values are expressed as mean \pm SEM. Statistical analysis: unpaired Student's $t$ test. 
moted SMC proliferation, increased the percentage of cells in S phase, and increased migration from $\mathrm{Fn}_{-} \mathrm{EDA}^{-/-} \mathrm{Apoe}^{-/}$, but not from Fn-EDA ${ }^{-/-} \mathrm{TLR}^{-/-}$Apoe $^{-/}$, mice (Figure 6, B-D). Furthermore, exogenous $\mathrm{cFn}$ potentiated TLR4-dependent Akt1/mTOR signaling when compared with pFn (Figure 7A). Concomitantly, TNF- $\alpha$ and IL-1 $\beta$ secretion levels were significantly increased in cFn-treated SMCs from Fn-EDA ${ }^{-/-}$Apoe $^{-/-}$, but not from Fn-EDA ${ }^{-1-}$ $\mathrm{TLR}^{-/-} \mathrm{Apoe}^{-/-}$, mice (Figure 7B). Because Akt is required for SMC proliferation and migration (29), we determined whether Akt contributes to $\mathrm{CFn}$-mediated SMC proliferation and migration. Aortic SMCs from Fn-EDA ${ }^{-/-} \mathrm{Apoe}^{-/-}$and Fn-EDA ${ }^{-/-} \mathrm{TLR}^{-/-} \mathrm{Apoe}^{-/-}$mice were treated with $\mathrm{cFn}$ in the presence of the PI3K-specific inhibitor LY294002, which is known to inhibit Akt-dependent cell proliferation and migration. We found that LY294002 partially inhibited exogenous $\mathrm{cFn}$-mediated cell proliferation and migration in the aortic SMCs from Fn-EDA ${ }^{-/}$Apoe $^{-/-}$mice. Although, LY294002 treatment in SMCs of Fn-EDA ${ }^{-/-} \mathrm{TLR}_{4}^{-/-} \mathrm{Apoe}^{-/-}$mice resulted into a further decrease in cell proliferation and migration, it was not significant (Figure 7C). Together, these results suggest that Akt1 signaling contributes in part to Fn-EDA/TLR4-mediated SMC proliferation and migration in vitro. Since both TLR4 and integrin signaling may lead to activation of ERK and NF- $\mathrm{KB}(15,31)$, we determined the role of ERK in $\mathrm{CFn}$-mediated SMC proliferation and migration. Stimulation of SMCs from Fn-EDA ${ }^{-/} \mathrm{Apoe}^{-/-}$mice with $\mathrm{CFn}$ caused a rapid and transient increase in ERK phosphorylation that reached a maximum at 30 minutes (Supplemental Figure 9A). Pretreatment of SMCs from Fn-EDA ${ }^{-/} \mathrm{Apoe}^{-/-}$mice with the ERK inhibitor U0126 (10 $\mu \mathrm{M}$ for 30 minutes) partially inhibited cFn-induced SMC proliferation and migration. Although ERK inhibition in the SMCs of Fn-EDA ${ }^{-/-} \mathrm{TLR}^{-/-} \mathrm{Apoe}^{-/-}$mice resulted in a further decrease in SMC proliferation and migration, it did not reach baseline, suggesting that besides TLR4 and ERK, other signaling pathways may be involved in $\mathrm{CFn}$-induced SMC proliferation and migration (Supplemental Figure 9B). Together, these results demonstrate roles for both integrin and TLR4 in Fn-EDAmediated SMC proliferation and migration.

TLR4 contributes to Fn-EDA-mediated neointimal hyperplasia and SMC proliferation. We targeted TLR4 in vivo because of the evidence that TLR4 contributes to Fn-EDA-mediated inflammation and atherosclerosis progression (17, 32). Additionally, TLR4 deletion had a more pronounced effect on SMC proliferation and migration (1.2 fold) when compared with RGDS peptide. Susceptibility to neointimal hyperplasia was studied at 28 days following wire injury in the carotid arteries of Apoe ${ }^{-/-}$and Fn-EDA ${ }^{-/-} \mathrm{Apoe}^{-/-}$mice on a TLR4-deficient background. Controls included $\mathrm{Apoe}^{-/-}$and Fn-EDA ${ }^{-/-} \mathrm{Apoe}^{-/-}$littermates. Both male and female mice were used to determine sex-based differences. Deletion of TLR4 in Apoe ${ }^{-/-}$mice resulted in a marked ( $\left.50 \%\right)$ decrease in the neointimal area and the neointimal/medial area ratio $\left(P<0.05\right.$ vs. Apoe ${ }^{-/-}$mice; Figure $\left.8 \mathrm{~A}\right)$. No sex-based differences were observed. Body weights and total cholesterol levels were comparable among groups (not shown). No significant differences in the neointimal area or neointimal/medial area ratio were observed between Fn-EDA ${ }^{-/-} \mathrm{TLR}^{-/-} \mathrm{Apoe}^{-/-}$mice and control Fn-EDA ${ }^{-/-}$Apoe $^{-/-}$mice (Figure 8A). Since the decrease in the neointimal area could be due to decreased neointimal cell proliferation or increased apoptosis, we analyzed SMC proliferation and apoptosis in wire-injured carotid artery sections. We found that Fn-EDA promoted TLR4-dependent SMC proliferation but did not affect apoptosis (Figure 8, B and C). In parallel, we confirmed these findings in vitro using PDGF-BB-stimulated aortic SMCs. Significant decreases in BrdU incorporation, percentage of cells in S phase, and migration were observed in SMCs of TLR4 ${ }^{-/-}$ Apoe $^{-/-}$mice when compared with Apoe ${ }^{-/-}$mice $(P<0.05$; Supplemental Figures 10 and 11). BrdU incorporation, percentage of cells in $\mathrm{S}$ phase, and migration were comparable between $\mathrm{Fn}-\mathrm{EDA}^{-/-}$ $\mathrm{TLR}^{-/-} \mathrm{Apoe}^{-/-}$and $\mathrm{EDA}^{-/-} \mathrm{Apoe}^{-/-}$mice (Supplemental Figures 10 and 11). Together, these results suggest that TLR4 contributes to Fn-EDA-mediated SMC proliferation and migration, and thereby promotes neointimal hyperplasia in the comorbid condition of hyperlipidemia.

SMC-derived Fn-EDA potentiates phenotypic switching and intimal hyperplasia. To elucidate the specific role of SMC-derived Fn-EDA in the neointimal hyperplasia and phenotypic switching in the comorbid condition of hyperlipidemia, we generated SMC-specific Fn-EDA-null mice on an Apoe-deficient background (Fn-EDA ${ }^{\mathrm{fl} / \mathrm{ll}} \mathrm{SM} 22 \alpha \mathrm{Cre}^{+} \mathrm{Apoe}^{-/-}$; Supplemental Figure 12A). Genomic PCR confirmed the presence of the SM22 $\alpha$ Cre gene in Fn-EDA ${ }^{\mathrm{fl} / \mathrm{ll}} \mathrm{Apoe}^{-/-}$mice (Supplemental Figure 12B). Immunostaining revealed Fn-EDA expression within neointimal SMCs in EDA ${ }^{\mathrm{f} / \mathrm{fl}}$ Apoe $^{-/-}$mice, whereas Fn-EDA expression was minimal and was mainly restricted to the luminal area in Fn-EDA ${ }^{\mathrm{f} / \mathrm{fl}} \mathrm{SM} 22 \alpha \mathrm{Cre}^{+}$ Apoe $^{-/-}$mice (Supplemental Figure 13). qPCR confirmed the absence of Fn-EDA mRNA in SMCs but not in endothelial cells or hepatocytes of Fn-EDA ${ }^{\mathrm{f} / \mathrm{fl}} \mathrm{SM} 22 \alpha \mathrm{Cre}^{+} \mathrm{Apoe}^{-/-}$mutant mice (Figure 9A). Fn-EDA ${ }^{\mathrm{f} / \mathrm{fl}} \mathrm{Apoe}^{-/-}$mice constitutively express Fn-EDA in plasma and all tissues (9). Intimal hyperplasia was comparable between $\mathrm{Apoe}^{-/-}$and Fn-EDA ${ }^{\mathrm{f} / \mathrm{fl}} \mathrm{Apoe}^{-/-}$mice. Furthermore, in vitro assays revealed that proliferation, percentage of cells in $S$ phase, and migration were comparable between aortic SMCs from $\mathrm{Apoe}^{-/-}$and Fn-EDA ${ }^{\mathrm{fl} / \mathrm{fl}} \mathrm{Apoe}^{-/-}$mice (Supplemental Figure 14, $A-D)$. Together, these results suggest that the constitutive expression of Fn-EDA in plasma and tissues does not further promote SMC proliferation and migration, or intimal hyperplasia. These results suggested that $\mathrm{Fn}-\mathrm{EDA}^{\mathrm{fl} / \mathrm{fl}} \mathrm{Apoe}^{-/-}$mice can be used as an appropriate control to define the SMC-specific role of Fn-EDA in intimal hyperplasia.

Susceptibility to neointimal hyperplasia was studied at 28 days following wire injury in the carotid artery of littermate Fn-EDA ${ }^{\mathrm{fl} / \mathrm{fl}} \mathrm{Apoe}^{-/-}$and Fn-EDA ${ }^{\mathrm{fl} / \mathrm{fl}} \mathrm{SM} 22 \alpha \mathrm{Cre}^{+} \mathrm{Apoe}^{-/-}$mice. Male and female mice were used to determine sex-based differences. Fn-EDA ${ }^{\mathrm{fl} / \mathrm{ll}} \mathrm{SM} 22 \alpha \mathrm{Cre}^{+} \mathrm{Apoe}^{-/-}$mice exhibited a marked ( $50 \%$ ) decrease in neointimal area as well as neointimal/medial ratio $(P$ $<0.05$ vs. Fn-EDA ${ }^{\mathrm{f} / \mathrm{fl}}$ Apoe $^{-/}$; Figure 9B). No sex-based differences were observed. Body weights were comparable among groups (not shown). Immunostaining revealed an increase in contractile proteins (SM22 $\alpha$ and SM-MHC) and a decrease in synthetic proteins (vimentin and osteopontin) in the neointima of $\mathrm{EDA}^{\mathrm{fl} / \mathrm{fl}}$ SM $22 \alpha \mathrm{Cre}^{+}$Apoe $^{-/-}$mice $\left(P<0.05\right.$ vs. Fn-EDA ${ }^{\mathrm{fl} / \mathrm{fl}} \mathrm{Apoe}^{-/-}$; Figure 9C). Next, we investigated whether deletion of Fn-EDA specifically in SMCs suppresses Akt1/mTOR signaling in vivo. We found that phospho-Akt1, phospho-mTOR, and phospho-NF- $\kappa \mathrm{B}$ were decreased in the homogenates prepared at 28 days after carotid artery injury in Fn-EDA ${ }^{\mathrm{fl} / \mathrm{fl}} \mathrm{SM} 22 \alpha \mathrm{Cre}^{+} \mathrm{Apoe}^{-/-}$mice $(P<0.05$ 
A

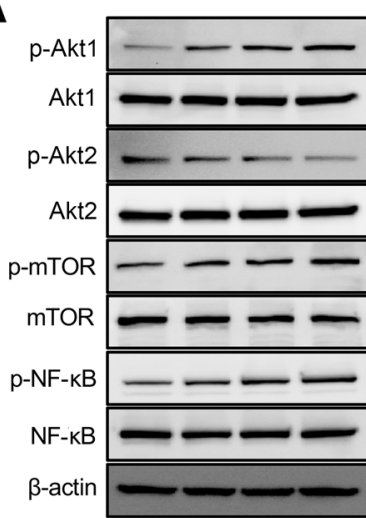

$\mathrm{cFn}(\mu \mathrm{g} / \mathrm{ml}) \quad 0 \quad 10 \quad 20 \quad 50$

Fn-EDA ${ }^{-1-\text { Apoe }^{-/}}$

C
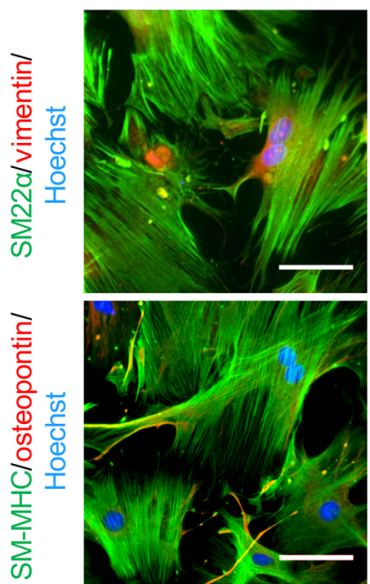

$\mathrm{pFn}$
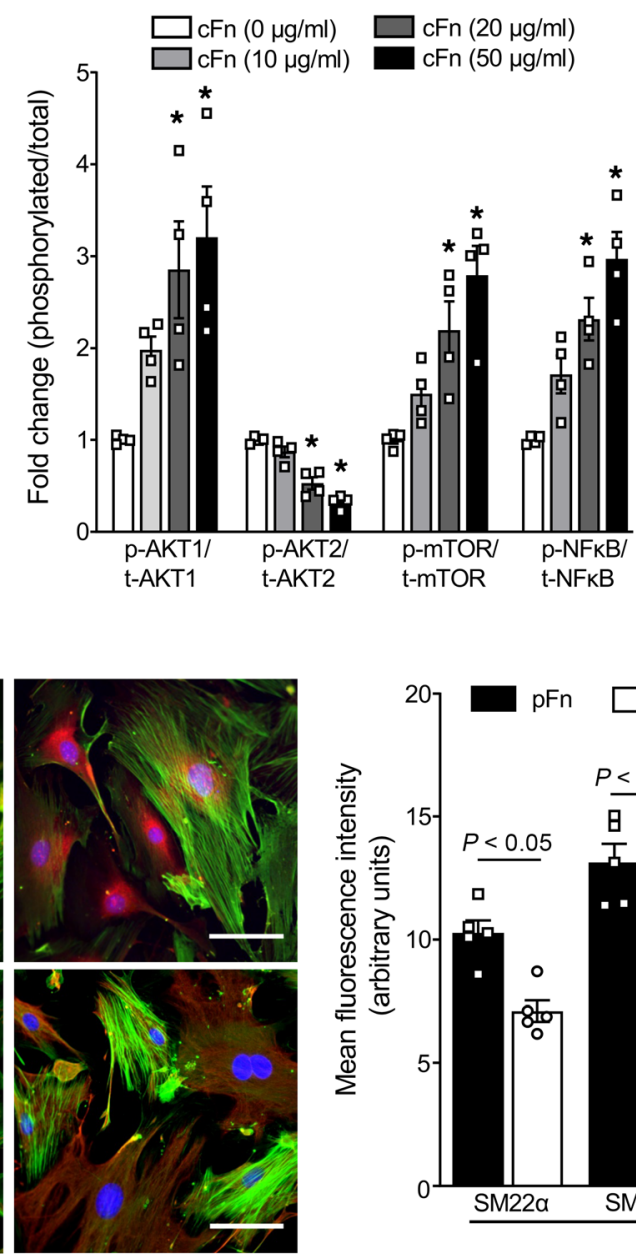

$\mathrm{cFn}$

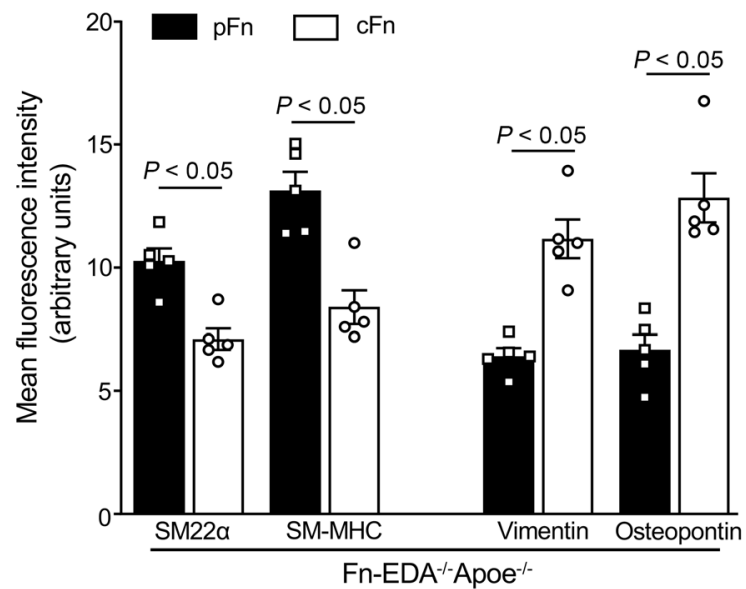

Fn-EDA ${ }^{--}$Apoe $^{-/}$
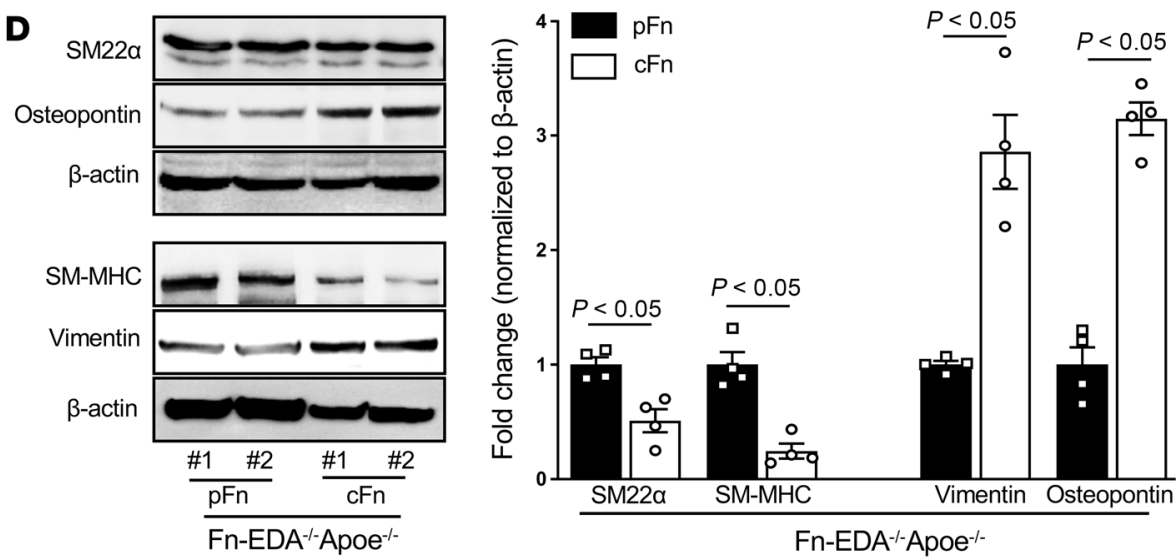

Figure 4. Exogenous cFn-EDA potentiates Akt1 signaling and promotes phenotypic switching. Quiescent aortic SMCs from Fn-EDA ${ }^{-/-}$Apoe ${ }^{-/-}$mice were stimulated with cellular Fn containing EDA (cFn-EDA; 0-50 $\mu \mathrm{g} / \mathrm{mL}$ ) for 24 hours. (A) Duplicate samples were run in the same gels, with the membrane cut in half, and then probed for total and phosphorylated proteins separately. Representative Western blots and densitometric analysis showing the dose-dependent effect of cFn-EDA on Akt signaling components ( $n=4$ per group). (B) ELISA quantification of TNF- $\alpha$ and IL-1 $\beta$ in supernatant medium from cFn-EDA $\left(20 \mu \mathrm{g} / \mathrm{mL}\right.$ ) treated SMCs ( $n=4$ per group). (C) Quiescent aortic SMCs from Fn-EDA ${ }^{/-}$Apoe ${ }^{-/-}$mice were stimulated with either $\mathrm{cFn}$-EDA (20 $\left.\mu \mathrm{g} / \mathrm{mL}\right)$ or $\mathrm{pFn}$ lacking EDA ( $20 \mu \mathrm{g} / \mathrm{mL}$ ). The left panels show representative immunostaining images for the contractile proteins SM22 $\alpha$ (green) and SM-MHC (green), and the synthetic proteins vimentin (red) and osteopontin (red). Scale bars: $25 \mu \mathrm{m}$. The right panel shows quantification of the immunostaining for SM $22 \alpha$, SM-MHC, vimentin, and osteopontin ( $n=4$ per group). (D) Representative immunoblots and densitometric analysis of SM22 $\alpha$, SM-MHC, vimentin, and osteopontin in aortic SMCs from Fn-EDA ${ }^{-/-}$Apoe ${ }^{-/-}$mice stimulated with either cFn-EDA (20 $\mu \mathrm{g} / \mathrm{mL}$ ) or pFn lacking EDA (20 $\left.\mu \mathrm{g} / \mathrm{mL}\right)(n=4 \mathrm{per}$ group). Blots for SM $22 \alpha$ and osteopontin are from the same biological sample, and blots for SM-MHC and vimentin are from another biological sample. Values are represented as mean \pm SEM. Statistical analysis: (A and B) 1-way ANOVA with Bonferroni's post hoc test; (C and $\mathbf{D})$ unpaired Student's $t$ test. ${ }^{*} P<0.05$ vs. $c$ Fn ( $\left.0 \mu g / m L\right)$. 
vs. Fn-EDA ${ }^{\mathrm{fl} / \mathrm{fl}} \mathrm{Apoe}^{-/-} ;$Figure 9D). In addition to SMCs, stressed or stimulated endothelial cells express Fn-EDA (33). Indeed, we found luminal staining of Fn-EDA in the SMC-specific Fn-EDA deficient mouse (Supplemental Figure 13). Using endothelial-specific Fn-EDA-deficient mice (Fn-EDA ${ }^{\mathrm{f} / \mathrm{fl}} \mathrm{Tie} 2 \mathrm{Cre}^{+} \mathrm{Apoe}^{-/}$), we further evaluated the role of endothelial cell-derived Fn-EDA in the neointimal hyperplasia. Complete blood counts were similar to those in Fn-EDA ${ }^{\mathrm{f} / \mathrm{fl}} \mathrm{Apoe}^{-/-}$mice (Supplemental Table 2). qPCR confirmed the absence of Fn-EDA mRNA in endothelial cells but not in hepatocytes or SMCs of Fn-EDA ${ }^{\mathrm{fl} / \mathrm{fl}} \mathrm{Tie}^{\mathrm{C}} \mathrm{Cre}^{+} \mathrm{Apoe}^{-/-}$mutant mice (Supplemental Figure 15A). To our surprise, we found that neointimal hyperplasia was comparable in the Fn-EDA ${ }^{\mathrm{f} / \mathrm{fl}}$ Tie$2 \mathrm{Cre}^{+} \mathrm{Apoe}^{-/-}$mice and Fn-EDA ${ }^{\mathrm{fl} / \mathrm{fl}} \mathrm{Apoe}^{-/-}$mice (Supplemental Figure 15B).

Targeting Fn-EDA with specific inhibitor suppresses aortic human SMC syntheticphenotype and inhibits proliferation. We first assessed the expression of Fn-EDA in human aortic SMCs (HASMCs). Using immunostaining, we found that HASMCs express Fn-EDA upon PDGF-BB stimulation (Figure 10A). We next evaluated the therapeutic potential of targeting Fn-EDA using specific antibodies against the EDA of Fn. Serum-deprived HASMCs were preincubated with anti-Fn-EDA Ig for 1 hour. Cells appeared normal during and after treatment. We found that mean fluorescence intensity of contractile proteins (SM22 $\alpha$ and SM-MHC) was increased whereas synthetic proteins (vimentin and osteopontin) were decreased in the anti-EDA Ig-treated HASMCs when compared with control Ig-treated HASMCs $(P<0.05$; Figure 10B). The results were confirmed in parallel in HASMC lysates by Western blotting (Figure 10C). Together, these findings suggest that Fn-EDA mediates SMC phenotypic switching in HASMCs. We found that HASMC proliferation and migration were significantly attenuated in antiFn-EDA Ig-treated HASMCs compared with control Ig-treated HASMCs (Figure 11A). Furthermore, we found a significant reduction in phospho-Akt1, phospho-mTOR, and phospho-NF-kB p65 intensity levels (by Western blot) in anti-Fn-EDA Ig-treated aortic HSMCs $(P<0.05$ vs. control Ig-treated aortic HSMCs; Figure 11B). These results suggest that Fn-EDA may modulate neointimal hyperplasia in injured human vessels by promoting SMC phenotypic modulation, proliferation, and migration via upregulation of the Akt1/mTOR signaling pathway (Figure 11C).

\section{Discussion}

Herein, using in vitro and in vivo models, we demonstrate that the ECM protein Fn-EDA contributes to the proliferation, migration, and phenotype switching of SMCs during neointimal hyperplasia. We believe that the findings of this study are novel and may have clinical significance for the following reasons: First, we demonstrated the expression of Fn-EDA in the vicinity of SMC-rich neointima and peri-strut areas in human coronary arteries with bare metal stents. Second, irrespective of sexual phenotype, we provide evidence, for the first time to our knowledge, that germline or SMC-specific deletion of Fn-EDA markedly reduces neointimal hyperplasia. Furthermore, we show that lack of Fn-EDA in SMCs results in reduced PDGF-BB-stimulated proliferation and migration and suppresses phenotypic switching. Third, mechanistically, we demonstrated that Fn-EDA mediates SMC proliferation and migration via both integrin and TLR4 signaling pathway.
Patients with comorbid conditions such as hyperlipidemia have increased risk for restenosis $(21,22)$. Fn-EDA is elevated explicitly during hyperlipidemia and found in the neointima (10, 34-36). In line with these observations, we found elevated Fn-EDA expression in the neointima of WT and hyperlipidemic Apoe ${ }^{-/-}$ mice following vascular injury. Our studies are in agreement with previous studies that have shown an abundance of Fn-EDA in the neointima of human and rat arteries $(3,10,37)$. Notably, no prior studies have examined how specific deletion of the ECM protein Fn-EDA affects neointimal hyperplasia. We show that germline deletion of Fn-EDA in either WT or Apoe - $^{-/}$mice suppresses intimal hyperplasia following vascular injury, suggesting that Fn-EDA promotes intimal hyperplasia. Neointimal hyperplasia involves interaction between platelets, inflammatory cells, SMCs, and endothelial cells. SMCs play a critical role in the progression of neointimal hyperplasia through proliferation, migration, and ECM deposition. Upon vascular injury and in response to growth factors including PDGF-BB and FGF, SMCs progress through DNA replication and mitosis in a regulated series of cell cycle events. The role of SMC-specific Fn-EDA in SMC proliferation has not been investigated previously, but a few studies have suggested that ECM rich in Fn-EDA promotes the proliferation, migration, and differentiation of embryonic stem cells (38) and fibroblasts (39). Herein, we report that Fn-EDA is a PDGF-inducible gene and contributes to gene expression changes in the synthetic SMCs. We demonstrated that PDGF-BB-stimulated aortic SMCs from Apoe ${ }^{-/-}$ mice lacking Fn-EDA exhibited reduced proliferation that was concomitant with a decrease in the percentage of cells in S phase and decreased migration. These results suggest that ECM rich in Fn-EDA mediates neointimal hyperplasia by promoting SMC proliferation and migration. Switching of SMCs from a contractile to a synthetic phenotype is a fundamental feature of the progression of intimal hyperplasia. We demonstrated that deletion of Fn-EDA in SMCs suppresses phenotypic switching, thereby tending to keep SMCs in a contractile state. These results are in agreement with an earlier report that Fn-EDA expression was associated with a synthetic SMC phenotype (10).

We also investigated the molecular mechanism by which Fn-EDA promotes SMC proliferation and migration, and thereby neointimal hyperplasia. pFn is known to modulate SMCs from a contractile to a synthetic phenotype via an integrin-dependent mechanism (26). Both $\mathrm{pFn}$ and $\mathrm{cFn}$ bind to integrins such as $\alpha_{5} \beta_{1}, \alpha_{3} \beta_{1}, \alpha_{v} \beta_{1}$, and $\alpha_{v} \beta_{3}$ expressed in proliferating SMCs. Indeed, RGDS peptide inhibited both $\mathrm{pFn}$ - and cFn-mediated SMC phenotype switching and proliferation, suggesting a mechanistic role for integrin signaling in Fn-mediated modulation of SMCs from contractile to synthetic phenotype. Despite RGDS inhibition, the extent of SMC proliferation and migration was significantly higher in cFn-stimulated SMCs when compared with pFn-stimulated cells, suggesting the involvement of other endogenous Fn-EDA receptors that are not recognized by RGDS. We hypothesized that the innate immune receptor TLR4, in addition to integrins, might contribute to Fn-EDAmediated SMC proliferation and migration based on the following observations. First, Fn-EDA is an endogenous ligand for TLR4 (13). Second, evidence from murine studies suggests that Fn-EDA promotes skin fibrosis, thrombosis, inflammation, and 


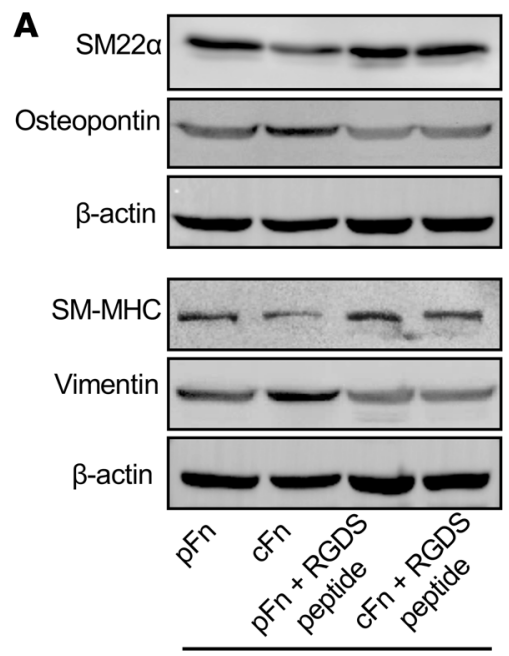

Fn-EDA-1-Apoe ${ }^{-1-}$

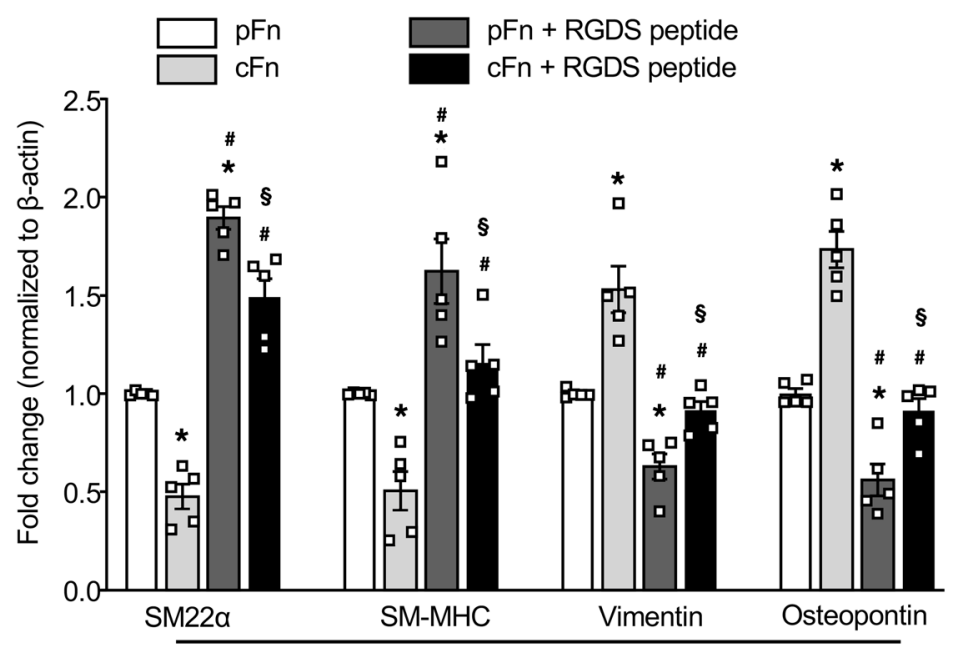

Fn-EDA ${ }^{-1-}$ Apoe $^{-1-}$

B

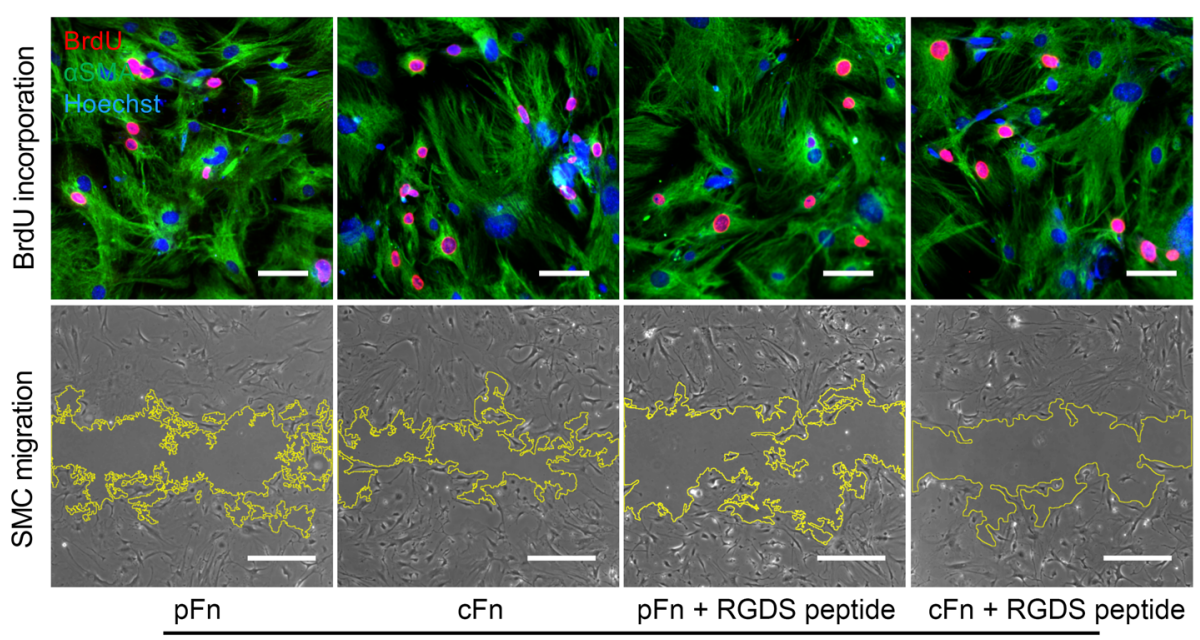

Fn-EDA-1-Apoe-1-

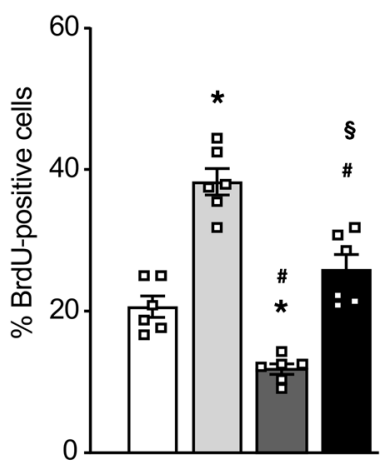

Fn-EDA ${ }^{-1-}$ Apoe $^{-/-}$

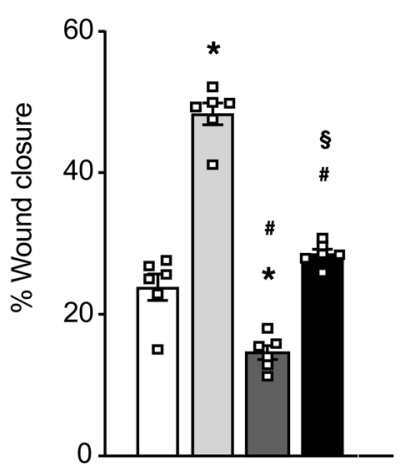

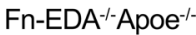

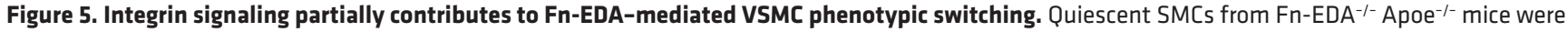
pretreated with RGDS-blocking peptide $(10 \mu \mathrm{M})$ for 30 minutes and then stimulated with either $\mathrm{cFn}$-EDA $(20 \mu \mathrm{g} / \mathrm{mL})$ or pFn lacking EDA $(20 \mu \mathrm{g} / \mathrm{mL})$ for 24 hours. (A) Cells were processed for Western blotting. Representative immunoblots and densitometric analysis of contractile proteins (SM22 $\alpha$, green; and SM-MHC, green) and synthetic proteins (vimentin, red; and osteopontin, red) ( $n=6$ per group). Blots for SM $22 \alpha$ and osteopontin are from the same biological sample, and blots for SM-MHC and vimentin are from another biological sample. (B) The top panels show representative images of SMC proliferation and migration. The bottom panels show quantification ( $n=6$ per group). Values are expressed as mean \pm SEM. Statistical analysis: 1-way ANOVA with Bonferroni's post hoc test. ${ }^{*} P<0.05$ vs. pFn-treated cells, ${ }^{\#} P<0.05$ vs. cFn-EDA-stimulated cells, ${ }^{\$} P<0.05$ vs. pFn + RGDS peptide-treated cells. Scale bars: BrdU assay, $50 \mu \mathrm{m}$; migration assay, $500 \mu \mathrm{m}$. 
A

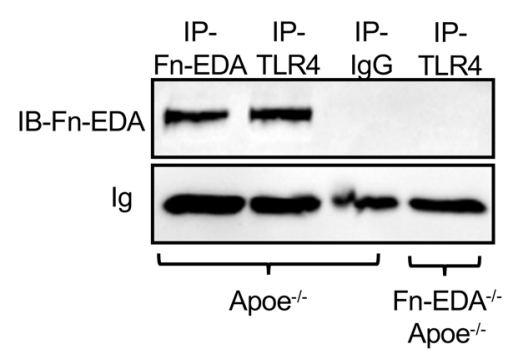

C

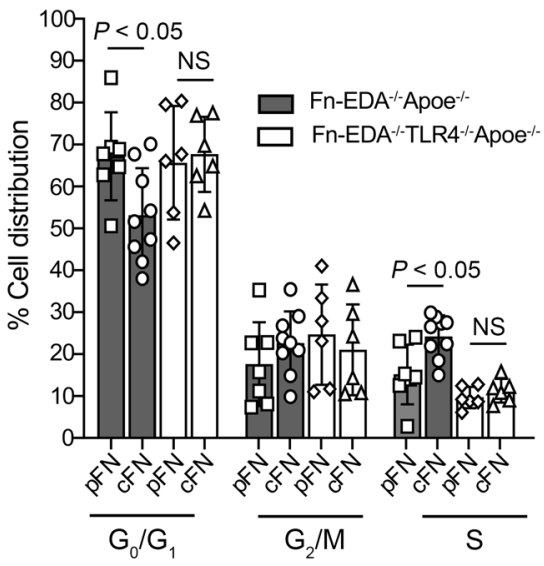

B

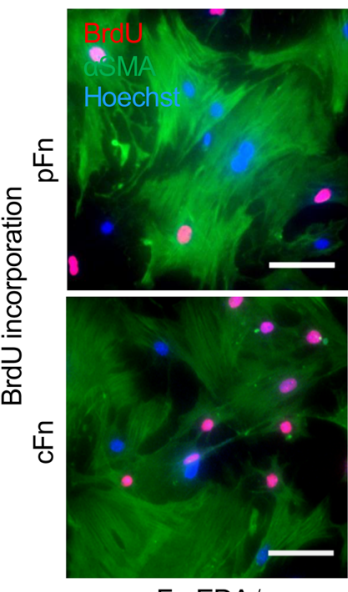

Fn-EDA-1/ Apoe $^{-1-}$

D

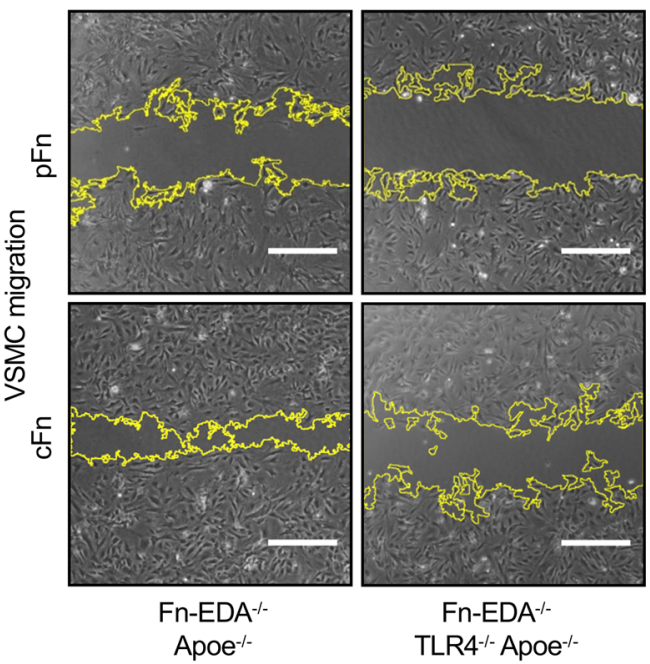

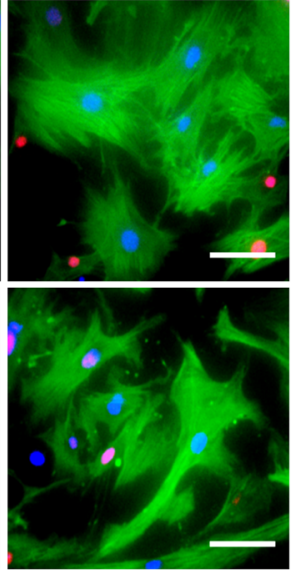

Fn-EDA-1-

$\mathrm{TLR}^{-1} \mathrm{Apoe}^{-1}$
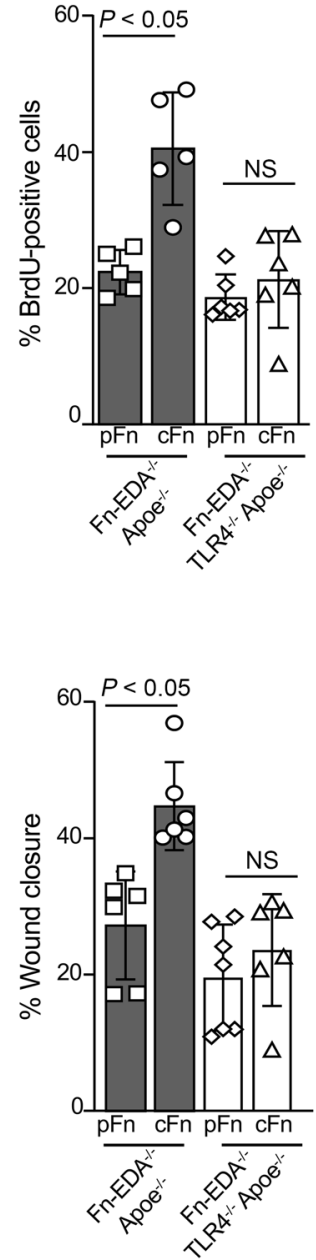

Figure 6. TLR4 contributes to Fn-EDA-mediated SMC proliferation and migration. (A) SMCs from Apoe ${ }^{-/-}$mice were stimulated with PDCF-BB for 24 hours, and cell extracts were immunoprecipitated (IP) with either anti-TLR4 or anti-Fn-EDA antibody and immunoblotted by anti-Fn-EDA. (B) Serumstarved aortic SMCs from Fn-EDA ${ }^{-1-} \mathrm{Apoe}^{-/-}$and Fn-EDA ${ }^{-/-} \mathrm{TLR}^{-/-}$Apoe $^{-/-}$mice were stimulated with either cFn-EDA (20 $\left.\mu \mathrm{g} / \mathrm{mL}\right)$ or pFn lacking EDA (20 $\mu \mathrm{g} / \mathrm{mL}$ ) for 24 hours. The left panels show representative BrdU-positive cells costained with $\alpha$ SMA (green) and Hoechst (blue). Scale bars: $50 \mu \mathrm{m}$. The right panel shows the quantification of BrdU-positive cells to the total number of cells ( $n=5-6$ per group). (C) Quantitative data of cell cycle distribution in aortic SMCs treated with either CFn-EDA or pFn lacking EDA ( $n=6-8$ per group). (D) The left panels show representative phase-contrast images of SMC migration in the scratch assay. The right panel shows quantification of the migrated area ( $n=6-7$ per group). Scale bars: $500 \mu \mathrm{m}$. Values are represented as mean \pm SEM. Statistical analysis: 1-way ANOVA with Bonferroni's post hoc test.

atherosclerosis via TLR4 signaling $(12,16-18,40)$. Third, TLR4 is present on SMCs and is known to promote SMC proliferation, migration, and neointimal hyperplasia (41). Herein, using in vitro and in vivo models, we showed that Fn-EDA interacts with TLR4 expressed on SMCs and mediates SMC proliferation, migration, and neointimal hyperplasia via TLR4. Notably, SMC proliferation, migration, and neointimal hyperplasia were comparable between Fn-EDA ${ }^{-/-} \mathrm{TLR}^{4^{-/}} \mathrm{Apoe}^{-/-}$mice and control Fn-EDA ${ }^{-/}$Apoe $^{-/}$mice. Together, the studies presented herein indicate that both integrin and TLR4 signaling pathways contribute to Fn-EDA-mediated SMC proliferation, migration, and intimal hyperplasia.

Next, we dissected the downstream pathway of integrin and TLR4 that contributes to Fn-EDA-mediated SMC proliferation, migration, and phenotypic switching. Evidence suggests that integrins activate FAK/Src signaling, which is known to potentiate cellular transduction pathways such as the MAPK and PI3K/ Akt pathways (42) that support cell proliferation and survival. Additionally, TLR4 mediates SMC proliferation via the PI3K/ Akt signaling pathway $(43,44)$. Three isoforms of Akt (Akt1, Akt2, and Akt3) are known to have different physiological functions (45). Akt1 is required for SMC proliferation and migration and is associated with the synthetic phenotype, whereas Akt2 exerts antiproliferative, antimigratory effects and promotes expression of contractile proteins and contractile morphology $(29,30)$. We found that Fn-EDA potentiated SMC proliferation and migration in vitro by upregulating phospho-ERK1/2 and phospho-Akt1 and downregulating phospho-Akt2. Furthermore, we found that the Fn-EDA/TLR4 axis promotes phosphorylation of the serine/threonine protein kinase mTOR concomitant with 
A

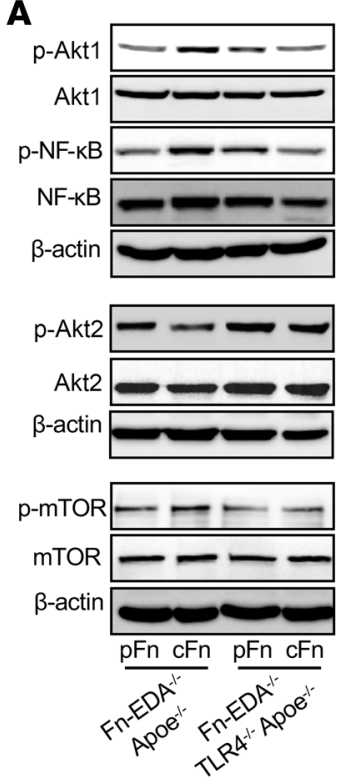

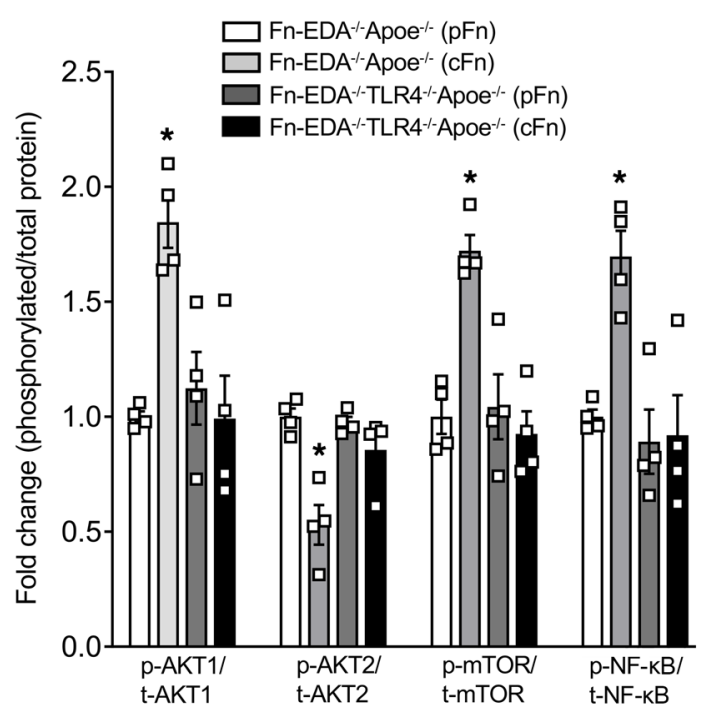

B

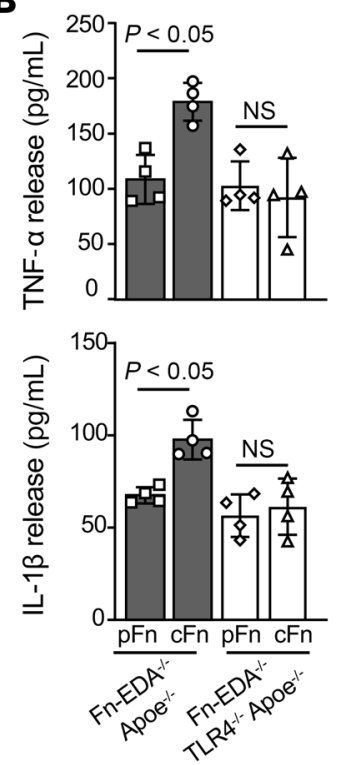

C

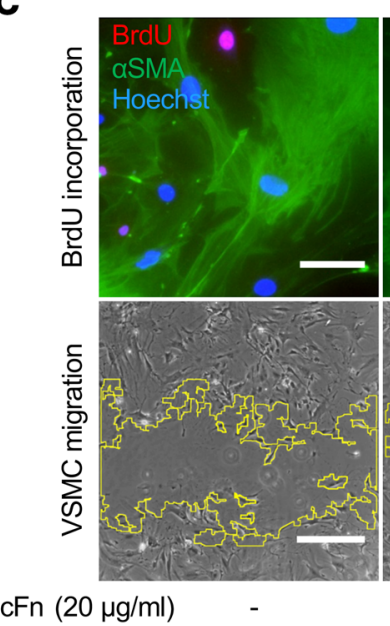

LY294002

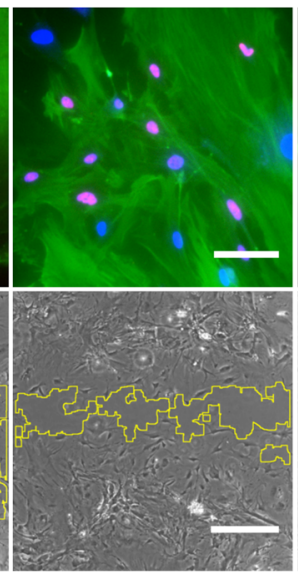

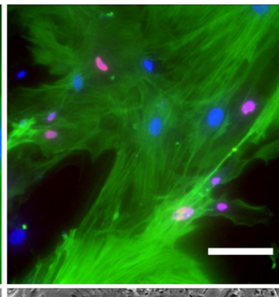
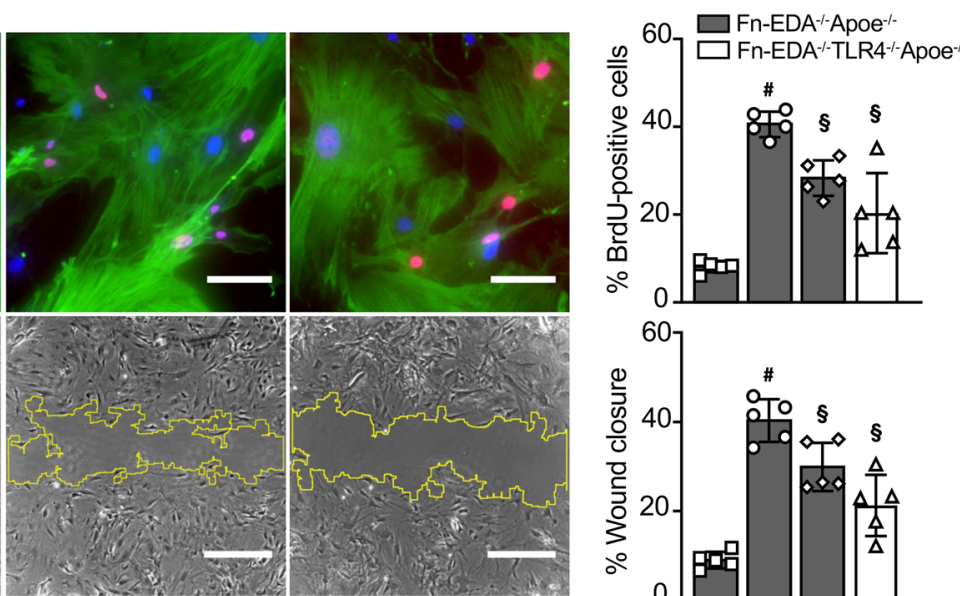

$+$

$+$

Fn-EDA'--Apoe ${ }^{-1-}$

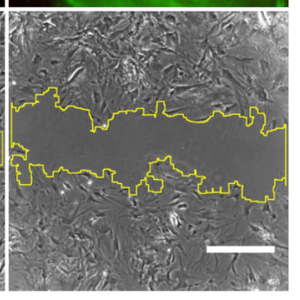

cFn $(20 \mu \mathrm{g} / \mathrm{ml})$ -

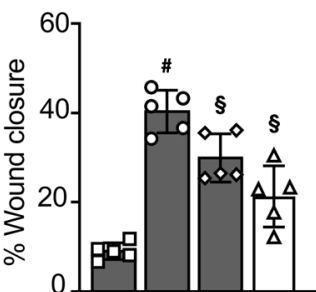

Y294002 - -+++

Figure 7. Exogenous Fn-EDA promotes TLR4-dependent Akt1/mTOR signaling. (A) Representative immunoblots and densitometric analysis of Akt signaling components in aortic SMCs stimulated with either cFn-EDA or pFn lacking EDA ( $n=4$ per group). Blots for Akt1 and NF- $\kappa B$ are from the same biological sample, and blots for Akt2 and mTOR are from another biological sample. (B) Quantification of TNF- $\alpha$ and IL-1 $\beta$ by ELISA ( $n=5$ per group). (C) Quiescent aortic SMCs from different groups were pretreated with Akt inhibitor (LY294002, $10 \mu \mathrm{M})$ and stimulated with either cFn-EDA (20 $\mu \mathrm{g} / \mathrm{mL})$ or pFn lacking EDA (20 $\mu \mathrm{g} / \mathrm{mL}$ ) for 24 hours. The left panels show representative images of SMC proliferation and migration. The right panels show quantification ( $n=5$ per group). Values are represented as mean \pm SEM. Statistical analysis: 1-way ANOVA with Bonferroni's post hoc test. ${ }^{*} P<0.05$ vs. pFn-treated Fn-EDA ${ }^{-1-}$ Apoe ${ }^{-/-}$cells,

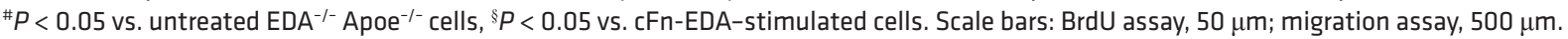

synthetic SMC phenotype with an increase in vimentin and osteopontin expression. mTOR is downstream of Akt, and inhibition of mTOR has been shown to promote contractile SMC phenotype with increased SM22 $\alpha$ expression (46). Multiple types of cells, including stressed endothelial cells, fibroblasts, macrophages, and platelets, express Fn-EDA $(33,47)$. Herein, using novel endothelial cell-specific and SMC-specific Fn-EDA-deficient mice, we provide evidence, for the first time to our knowledge, that SMC-derived Fn-EDA, but not endothelial-derived Fn-EDA, potentiates neointimal hyperplasia by promoting SMC migration and proliferation.
PCI with balloon angioplasty, often followed by stent implantation, is the most commonly performed procedure for the reopening of a stenosed artery. However, these procedures are associated with restenosis and other stent-related problems, including in-stent/scaffold thrombosis. We demonstrated the expression of Fn-EDA in the neointima and peri-strut areas in human coronary arteries with bare metal stents. To evaluate the potential clinical relevance of targeting Fn-EDA, we used human aortic SMCs pretreated with a specific Fn-EDA inhibitor, which suppressed the synthetic SMC phenotype and attenuated proliferation and migration. From these in vitro and in vivo studies, we propose a mechanistic 


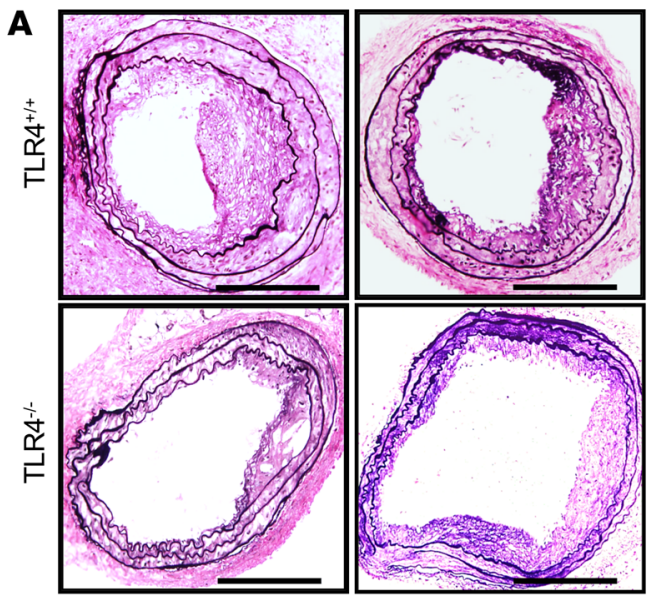

$\mathrm{Apoe}^{-/-}$

B

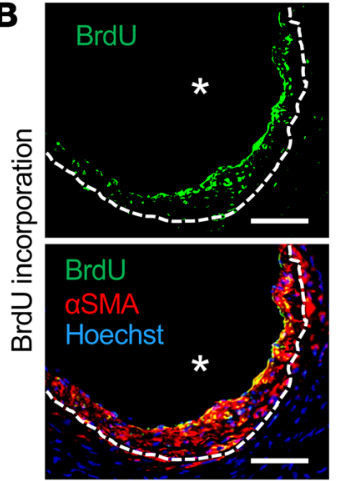

Apoe $^{-1-}$

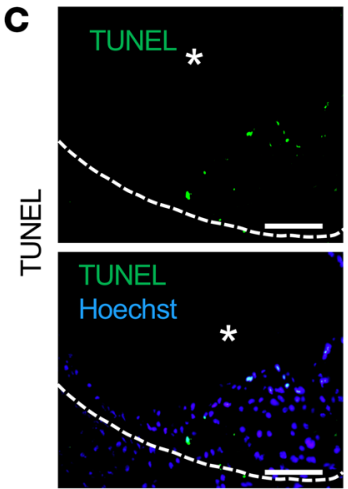

Apoe $^{-/-}$
Fn-EDA ${ }^{-/-} \mathrm{Apoe}^{-/-}$

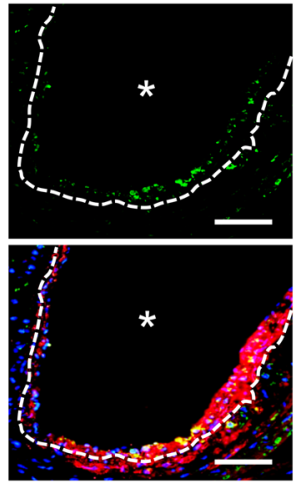

TLR4 ${ }^{-1-}$

Apoe $^{-/-}$
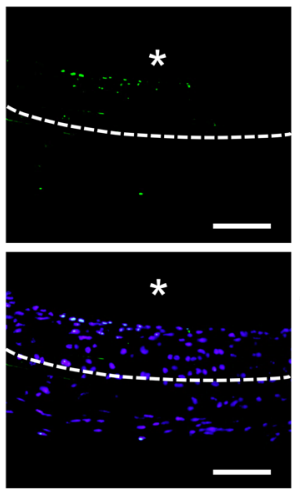

$\mathrm{TLR}^{-1-}$

Apoe $^{-1}$

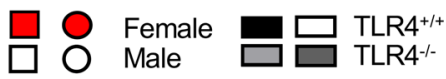
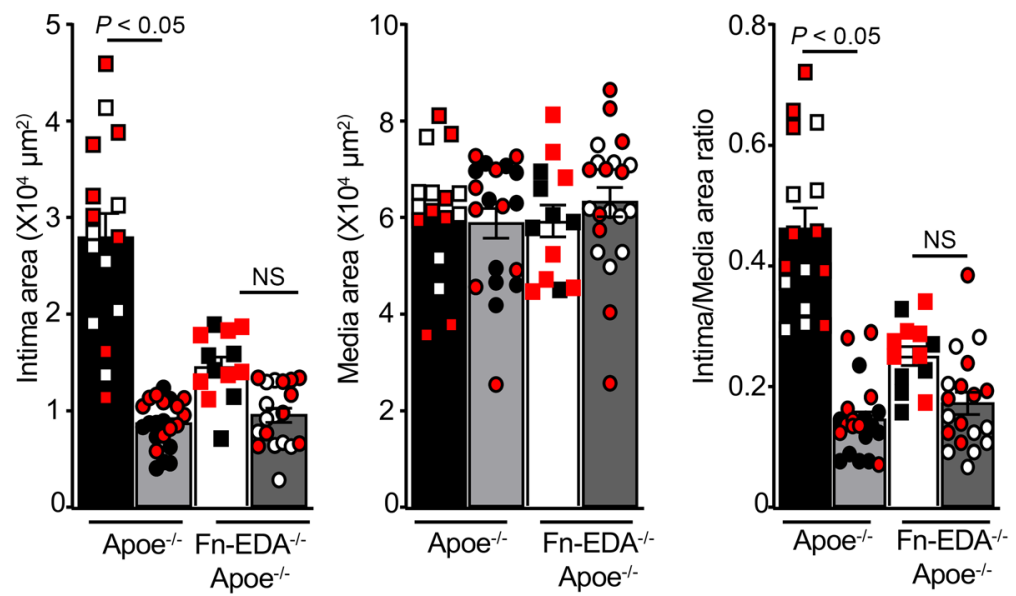

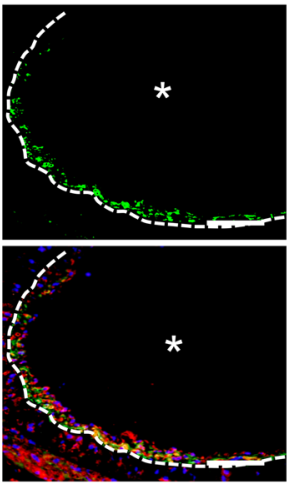

Fn-EDA-/- Apoe $^{-/-}$
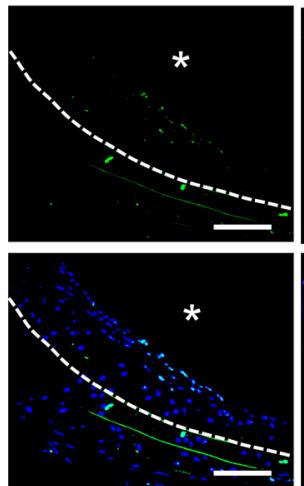

Fn-EDA-/- Apoe $^{-/-}$

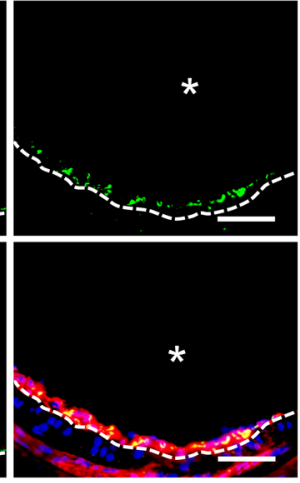

Fn-EDA $^{-1}$

TLR4 ${ }^{-1-\text { Apoe }^{-/-}}$
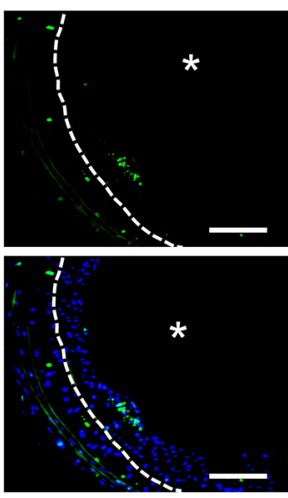

Fn-EDA $^{-1-}$

TLR4 ${ }^{-1-\text { Apoe }^{-1-}}$
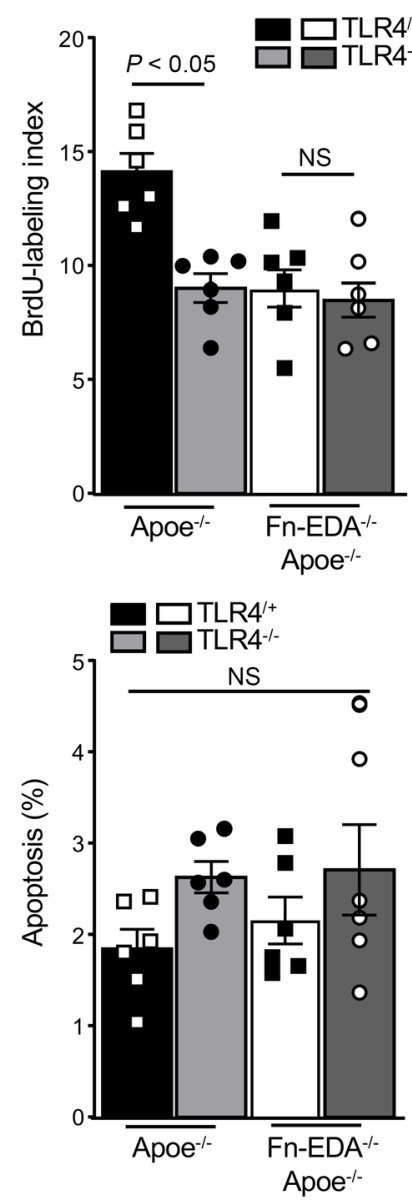

Figure 8. Fn-EDA promotes neointimal hyperplasia and SMC proliferation in vivo via TLR4. (A) The left panels show representative photomicrographs of Verhoeff's/van Gieson-stained carotid artery sections of each genotype mice after 28 days of injury ( $n=6-11$ per group). Scale bars: $200 \mu$ m. The right panels show quantification of intimal area, medial area, and a ratio of intimal to medial area. Each dot represents a single mouse. (B) The left panels show representative BrdU-positive cells (green) counterstained with aSMA (red) and Hoechst (blue). The right panel shows quantification of percent BrdU-positive cells. Scale bars: $100 \mu \mathrm{m} ; n=6$ per group. (C) The left panels show representative TUNEL-positive cells (green) counterstained with Hoechst (blue). The right panel shows the quantification of TUNEL-positive cells. Scale bars: $100 \mu \mathrm{m} ; n=6$ per group. Internal elastic lamina is circumscribed by dotted lines.

* Lumen side. Values are represented as mean \pm SEM. Statistical analysis: 1-way ANOVA with Bonferroni's post hoc test. 
A
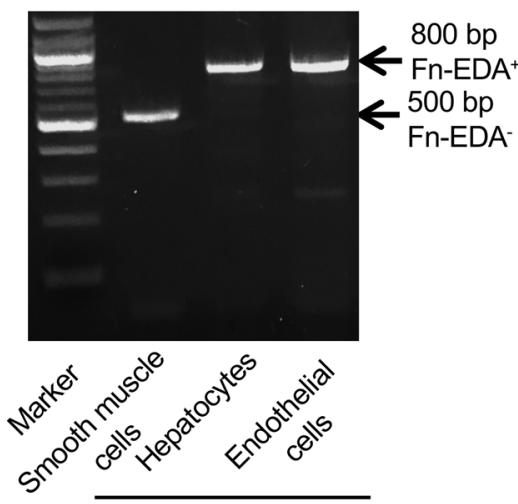

$\mathrm{Fn} \mathrm{EDA}^{\mathrm{fl} / \mathrm{fl}}$

SM22aCre ${ }^{+} \mathrm{Apoe}^{-/-}$
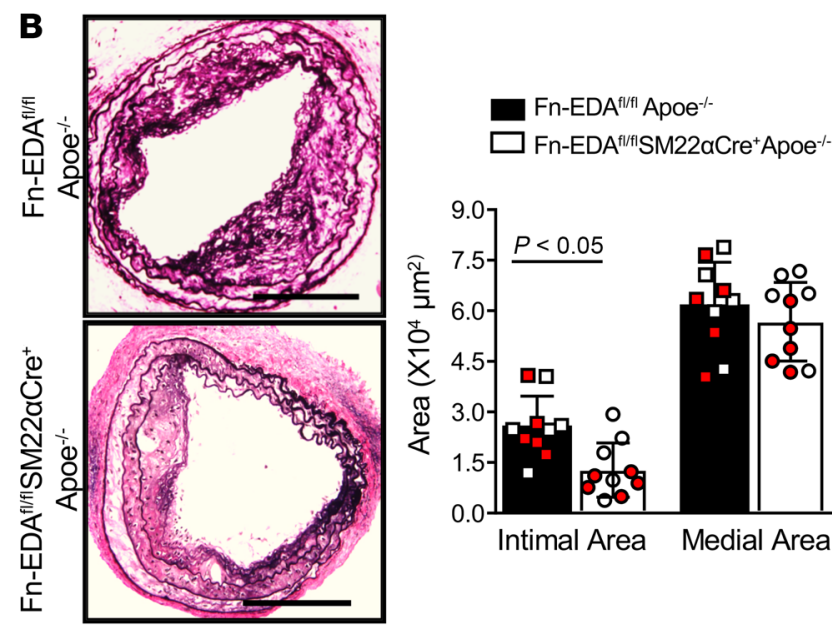

Fn-EDA ${ }^{\mathrm{Al} / \mathrm{fl}} \mathrm{Apoe}^{-1-}$

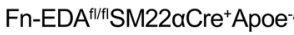

O Femal

$\square$ O Male

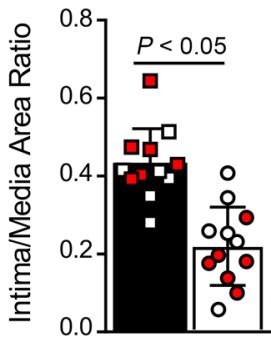

C
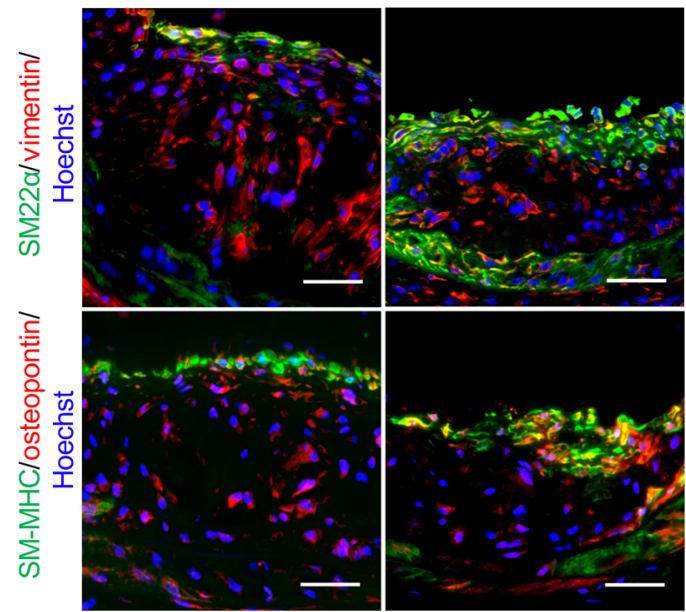

Fn-EDA fl/filApoe ${ }^{-/-}$

Fn-EDA ${ }^{\mathrm{f} / / 1 / \mathrm{SM} 22 \alpha \mathrm{Cre}{ }^{+}}$ Apoe $^{-/-}$

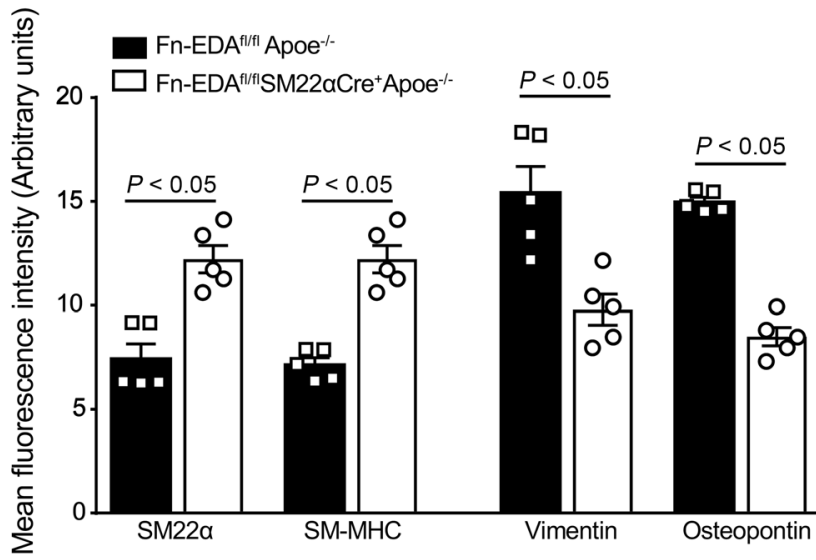

D
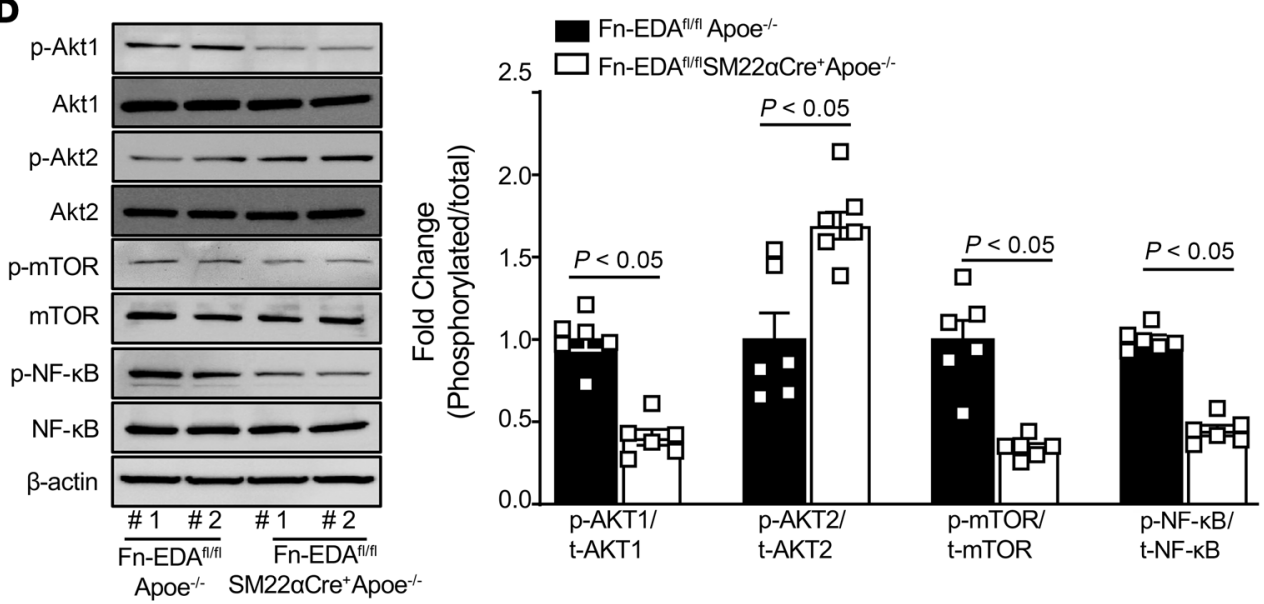

Figure 9. SMC-specific Fn-EDA mediates neointimal hyperplasia by promoting phenotypic switching. (A) RT-PCR confirmed the absence of Fn-EDA mRNA in SMCs but not in endothelial cells or hepatocytes of Fn-EDA $A^{\mathrm{fl} / f l} \mathrm{SM} 22 \alpha \mathrm{Cre}^{+} \mathrm{Apoe}^{-/-}$mice. (B) The left panels show representative photomicrographs of Verhoeff's/van Gieson-stained carotid artery sections of Fn-EDA ${ }^{\mathrm{fl} / f \mathrm{l}} \mathrm{Apoe}^{-/-}$and Fn-EDA ${ }^{\mathrm{fl} / \mathrm{fl}} \mathrm{SM} 22 \alpha \mathrm{Cre}^{+}$Apoe ${ }^{-/-}$mice after 28 days of injury $(n=5$ per group). Scale bars: $200 \mu \mathrm{m}$. The right panels (bar graphs) show quantification of intimal area and a ratio of intimal to medial area. Each dot represents a single mouse. (C) The left panels show representative SM22 $\alpha$-positive and vimentin-positive immunostaining in carotid artery sections after 28 days of injury. The right panel shows quantification ( $n=5$ per group). Scale bars: $50 \mu \mathrm{m}$. (D) Duplicate samples were run in the same gels, with the membrane cut in half, and then probed for total and phosphorylated proteins separately. Representative immunoblots and densitometric analysis of Akt and its downstream proteins in the carotid lysates 28 days after injury ( $n=6$ per group). \#1 and \#2 are two different samples. Each dot represents a single mouse. Values are expressed as mean \pm SEM. Statistical analysis: unpaired Student's $t$ test. 
model in which, following vascular injury, Fn-EDA synthesized by proliferative SMCs potentiates modulation of arterial SMCs from contractile to synthetic phenotype via both integrin and TLR4 signaling in an autocrine and/or paracrine feed-forward pathway. These signals may amplify the inflammatory microenvironment in the injury region, thereby mediating intimal hyperplasia. A summary of the proposed mechanism is provided in Figure 11C.

A particular strength of our study is the observation that Fn-EDA is expressed in the neointima of stented human coronary artery specimens and targeting Fn-EDA with a specific inhibitor suppressed the synthetic phenotype in cultured human aortic SMCs and markedly reduced SMC proliferation and migration. The studies, using several novel mutant mouse models, unequivocally support a causal connection between Fn-EDA synthesized by proliferating SMCs and neointimal hyperplasia exacerbation. These findings may have implications for treating post-angioplasty neointimal hyperplasia in patients at risk of atherosclerosis. Despite its strengths, our study has limitations, which include the use of a wire injury model that was chosen because it partially mimics balloon angioplasty or intraluminal stent placement. Further studies using authentic stent models will be required to confirm these findings. Extending these studies to large-animal models of the human disease also will be required to validate the translational feasibility of our current findings.

\section{Methods}

Animals. Eight- to ten-week-old male and female WT, Fn-EDA ${ }^{-/}$,

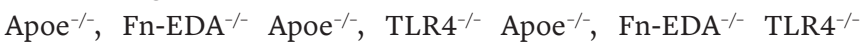
Apoe $^{-/-}$, and Fn-EDA ${ }^{\mathrm{fl} / \mathrm{fl}}$ Apoe $^{-/-}$mice on the C57BL/6J background, weighing around $22 \pm 2 \mathrm{~g}$, were used in this study. We have characterized and described these mice previously (40). Briefly, Fn-EDA ${ }^{-/-}$ mice and Fn-EDA ${ }^{\mathrm{fl} / \mathrm{fl}}$ mice (backcrossed $>15$ times to $\mathrm{C} 57 \mathrm{BL} / 6 \mathrm{~J}$ ) were crossed to Apoe ${ }^{-/-}$mice (The Jackson Laboratory) to generate

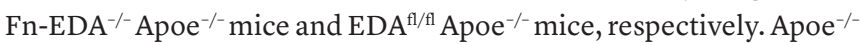
mice were crossed to TLR $4^{-/-}$mice to generate $\mathrm{TLR}_{4}^{-/-} \mathrm{Apoe}^{-/-}$mice. Fn-EDA ${ }^{-/-} \mathrm{Apoe}^{-/-}$mice were crossed to TLR4 ${ }^{-/-} \mathrm{Apoe}^{-/-}$mice to obtain Fn-EDA ${ }^{-/-}$TLR4 $^{-/-}$Apoe $^{-/-}$mice. To generate SMC-specific Fn-EDAdeficient mice on an Apoe-deficient background, first SM22 $\alpha \mathrm{Cre}^{+}$ mice were crossed with Apoe ${ }^{-/-}$mice to generate SM22 $\alpha \mathrm{Cre}^{+} \mathrm{Apoe}^{-/-}$ mice. In the second step, Fn-EDA ${ }^{\mathrm{fl} / \mathrm{fl}} \mathrm{Apoe}^{-/-}$mice were crossed to SM22 $\alpha \mathrm{Cre}^{+} \mathrm{Apoe}^{-/-}$mice to generate Fn-EDA ${ }^{\mathrm{f} / \mathrm{fl}} \mathrm{SM} 22 \alpha \mathrm{Cre}^{+} \mathrm{Apoe}^{-/-}$ mice. To generate endothelial cell-specific Fn-EDA-deficient mice $\left(\right.$ Apoe $\left.^{-/-} \mathrm{Fn}_{-E^{-}} \mathrm{ED}^{\mathrm{fl} / \mathrm{fl}} \mathrm{Tie} 2 \mathrm{Cre}^{+}\right), \mathrm{Apoe}^{-/-} \mathrm{Fn}-\mathrm{EDA}^{\mathrm{fl} / \mathrm{fl}}$ mice were crossed to the Apoe ${ }^{-/-}$Tie2Cre ${ }^{+}$mice. These mice were described previously (48). Mice were genotyped by PCR according to protocols from The Jackson laboratory and as described before (49).

Antibodies. A detail list of antibodies with catalog numbers and companies is provided in Supplemental Table 3.

Human stented coronary artery. Placement of bare metal stents induces neointimal proliferation that progresses through different phases of repair with time (23). Human coronary artery specimens with a bare metal stent implanted with duration between 60 and 120 days were selected from CVPath Institute Stent Registry. We selected this time period because this phase is characterized by active progression of neointima and SMC proliferation in bare metal stents (23). Samples were morphologically screened and were excluded if the neointima was destroyed or damaged. We identified 6 samples that had the same type of stent. Patient coronary artery disease status and stent characteristics are shown in Supplemental Table 1. Briefly, the stented artery segments were fixed in formalin, dehydrated in a graded series of ethanol, and embedded in methylmethacrylate polymer. After polymerization, 2- to 3-mm segments were sawed from each stent. Sections 4-6 $\mu \mathrm{m}$ thick were cut from each of the segments on a Leica RM2155 rotary microtome equipped with a tungsten carbide blade, mounted on slides, and stained with H\&E.

Mouse carotid artery injury model. Carotid artery wire injury was performed using a guide wire as described previously (50). Briefly, the left common carotid artery was exposed by creation of a midline neck incision. After exposure of the external carotid artery, an incision was made through which a guide wire (0.015-in. diameter; C-SF-15-15, Cook Medical LLC) was inserted $1 \mathrm{~cm}$ toward the aortic arch. The wire was pulled back to the carotid bifurcation in a rotating fashion. This procedure was repeated a total of 3 times to ensure endothelial damage. After retrieval of the wire, the external carotid artery was ligated with a 7-0 vicryl suture. The wound was closed with a subcuticular 7-0 vicryl suture, and animals were allowed to recover before returning to the cages. Carotid arteries ( $5.0 \mathrm{~mm}$ from the injury site) were harvested at 14 and 28 days after an injury from each group and processed for cryostat sectioning. Some mice received a subcutaneous dose of BrdU (100 mg/ $\mathrm{kg}$ ) at 12 hours and again at 1 hour before sacrifice (51).

Morphometric analysis. Serial cross sections were stained with Verhoeff's/van Gieson stain to measure the extent of neointimal hyperplasia. Bright-field images were acquired using an Olympus BX51 microscope equipped with $\mathrm{a} \times 20$ objective. Intimal area, medial area, and intimal/medial area ratio were calculated using ImageJ software (NIH). The intimal area was determined by subtraction of the lumen area from the area circumscribed by the internal elastic lamina traced on stained sections. The medial area was defined as the area between an external elastic lamina and internal elastic lamina. Intimal/medial area ratio was calculated by division of intimal area by medial area (52). Calculations were made using the mean value of 4-6 sections (each $100 \mu \mathrm{m}$ apart) from each mouse artery as described before. ImageJ software was used for quantification.

Mouse and human aortic SMC culture and treatment. Human primary aortic smooth muscle cells (HASMCs) were purchased from American Type Culture Collection (catalog PCS-100-012) and cultured in Smooth Muscle Cell Growth Basal Medium (catalog CC-3181, Lonza) containing recombinant human FGF basic $(5 \mathrm{ng} / \mathrm{mL})$, insulin $(5 \mu \mathrm{g} / \mathrm{mL}), \mathrm{EGF}(5 \mathrm{ng} / \mathrm{mL})$, and FBS (5\%). Murine primary vascular smooth muscle cells (VSMCs) were isolated from the thoracic aorta by enzymatic digestion technique and cultured in DMEM as described previously (53). Briefly, thoracic aorta was isolated, cleaned of adventitial tissue, and incubated with $1.0 \mathrm{mg} / \mathrm{mL}$ collagenase in DMEM at $37^{\circ} \mathrm{C}$ for 90 minutes. After incubating for 90 minutes at $37^{\circ} \mathrm{C}$, the cell suspension was passed through a $70-\mu \mathrm{m}$ restrainer, washed with DMEM, and plated onto 1 well of a 6-well culture plate. The cells were maintained at $37^{\circ} \mathrm{C}$ in a $5 \% \mathrm{CO}_{2}$ humidified incubator. Cells were trypsinized after 7 days and subcultured. All VSMCs used for experiments were between the third and fifth passages.

EDA-containing and EDA-lacking recombinant peptides. FN sequences containing (EDA + peptide) or lacking (EDA - peptide) the EDA segment were generated from full-length $\mathrm{cFn}$ and full-length $\mathrm{pFn}$ cDNAs, respectively, as described before (39) and were used at a concentration of $10 \mu \mathrm{g} / \mathrm{mL}$. 
A

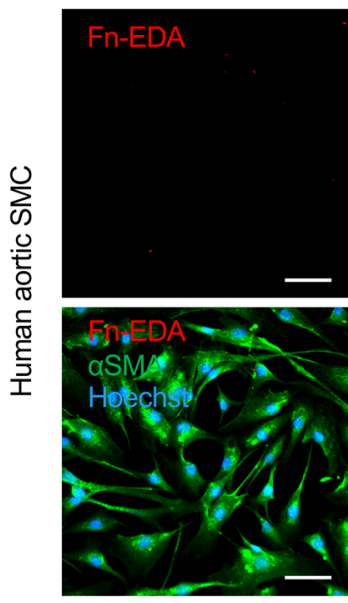

Quiescent

\section{B}

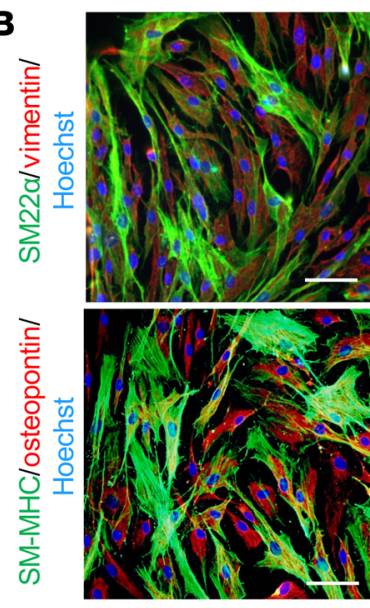

Quiescent
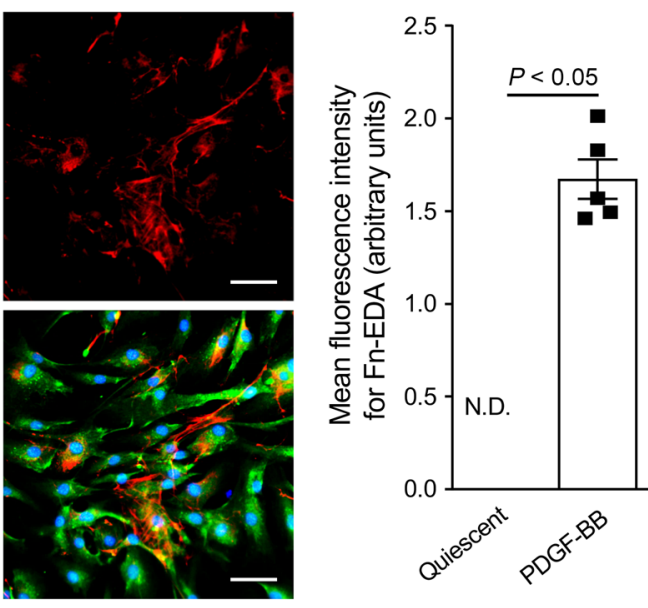

PDGF-BB
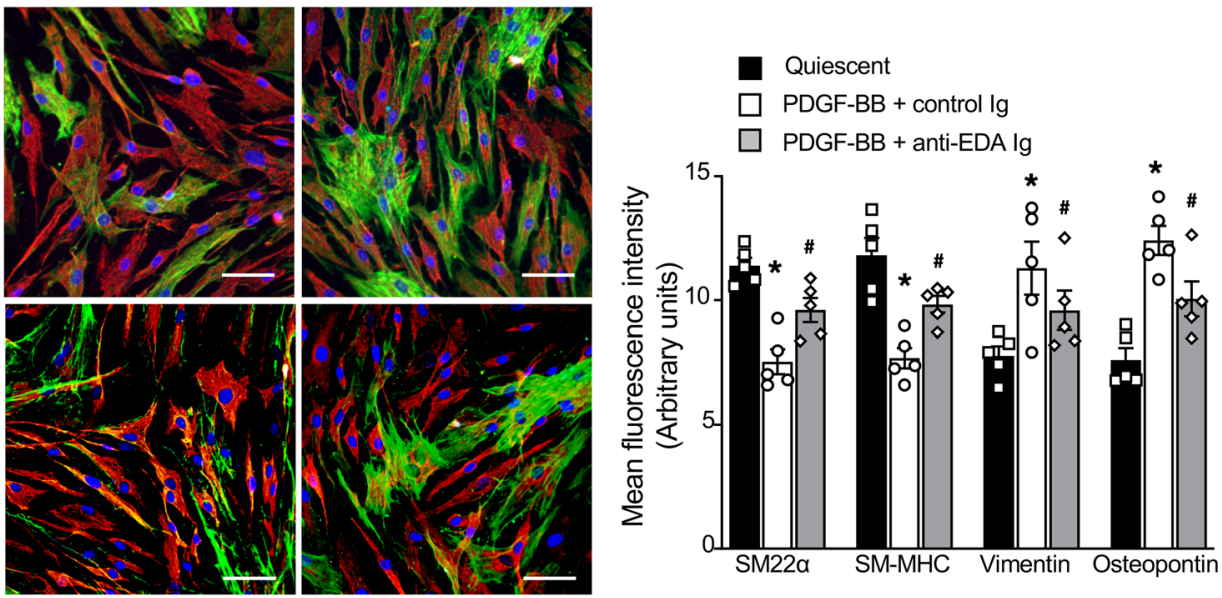

PDGF-BB + control Ig PDGF-BB + anti-EDA Ig
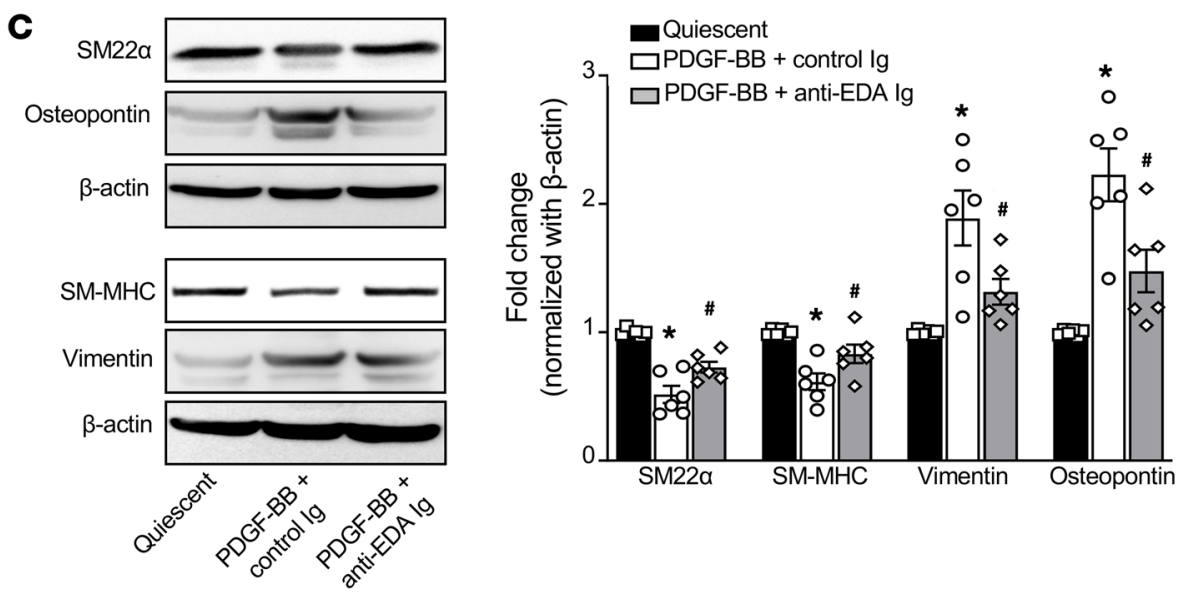

Figure 10. Targeting cFn with anti-Fn-EDA Ig suppresses synthetic phenotype and attenuates proliferation and migration of human aortic SMCs. (A) HASMC culture was serum-starved for 48 hours and stimulated with or without PDGF-BB for 24 hours. The left panels show representative double immunostaining for Fn-EDA (red) and SMC (green). The right panel shows the quantification of Fn-EDA ( $n=5$ per group). Scale bars: $50 \mu$ m; ND, not detected. (B) HASMC culture was pretreated with anti-EDA Ig for 1 hour before PDCF-BB stimulation. The left panels show representative immunostaining images for contractile proteins (SM22 $\alpha$, green; and SM-MHC, green) and synthetic proteins (vimentin, red; and osteopontin, red). Scale bars: $50 \mu \mathrm{m}$. The right panels show quantification of the fluorescence intensity for SM22 $\alpha$, SM-MHC, vimentin, and osteopontin ( $n=5$ per group). (C) Representative immunoblots and densitometric analysis of SM22 $\alpha, 5 M-M H C$, vimentin, and osteopontin ( $n=6$ per group). Blots for SM22 $\alpha$ and osteopontin are from the same biological sample, and blots for SM-MHC and vimentin are from another biological sample. Statistical analysis: (A) unpaired Student's $t$ test; (B and C) 1-way ANOVA with Bonferroni's post hoc test. ${ }^{*} P<0.05$ vs. quiescent, ${ }^{\#} P<0.05$ vs. PDGF-BB + control Ig. 
A

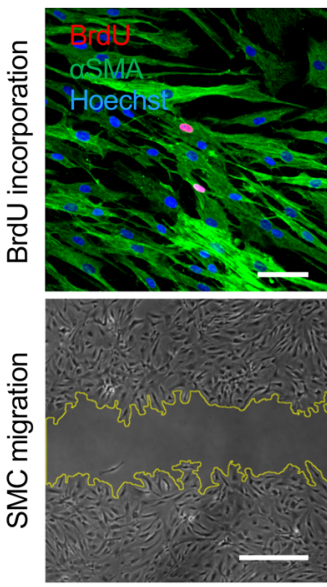

Quiescent

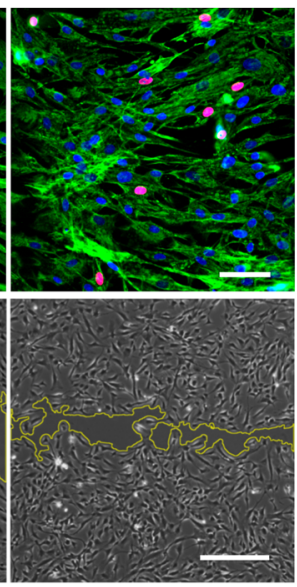

PDGF-BB + control Ig
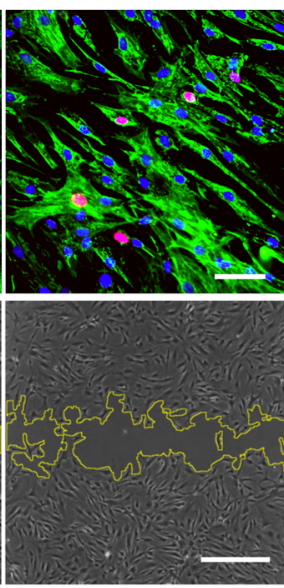

PDGF-BB + anti-EDA lg
B

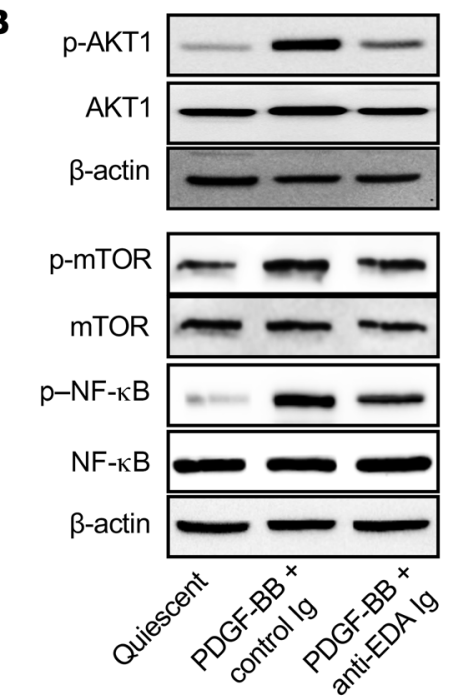

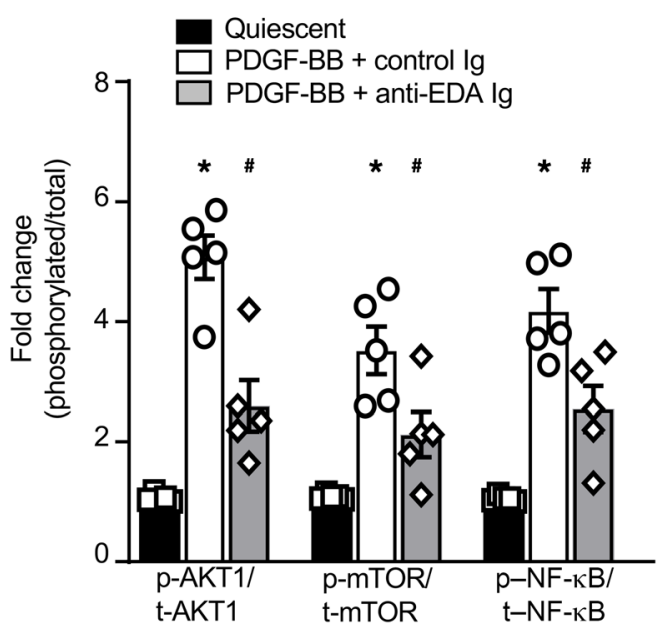

C

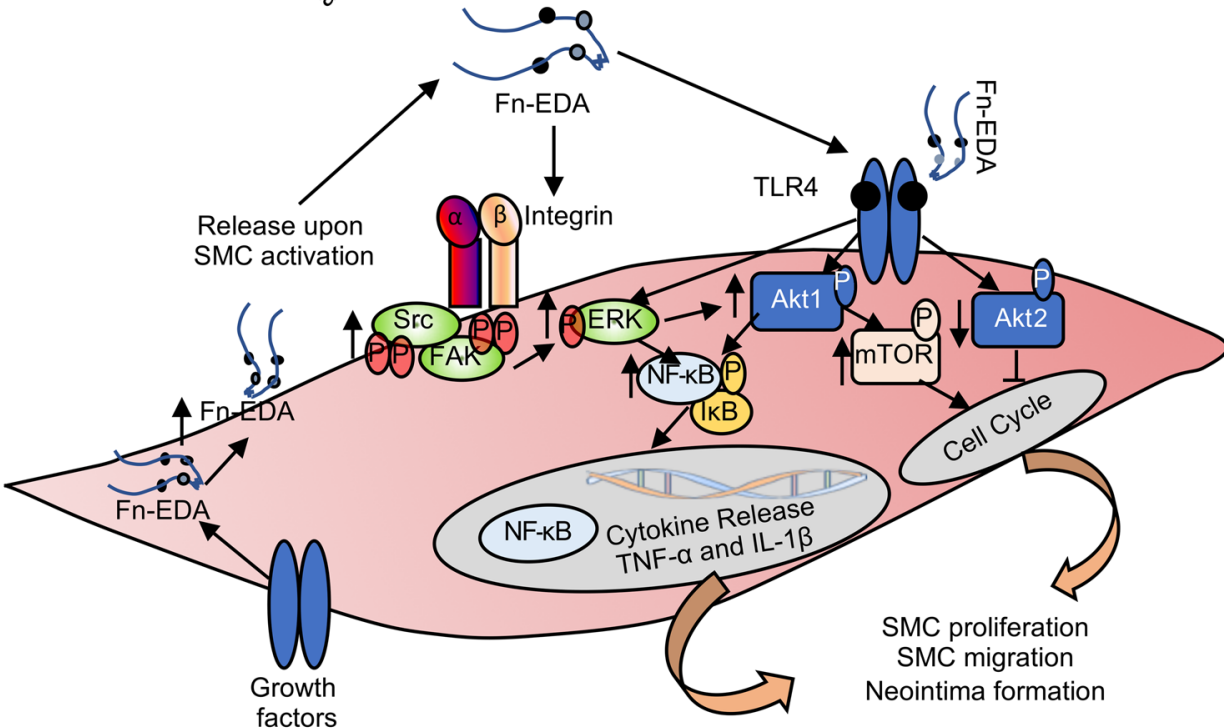

Figure 11. Targeting cFn with anti-Fn-EDA Ig attenuates proliferation and migration of human aortic SMCs. (A) The left panels show representative images of SMC proliferation and migration. The right panels show quantification ( $n=5-6$ per group). Scale bars: BrdU assay, $50 \mu \mathrm{m}$; migration assay, $500 \mu \mathrm{m}$. (B) Representative Western blots and densitometric analysis of Akt1, mTOR, NF- $\kappa B$, and $\beta$-actin ( $n=5$ per group). Blots for mTOR and NF- $\mathrm{KB}$ are from the same biological sample, and Akt1 is from another biological sample run in parallel. (C) Schematic showing the mechanism by which SMC-specific Fn-EDA mediates SMC proliferation, migration, and neointima hyperplasia. Statistical analysis: 1-way ANOVA with Bonferroni's post hoc test. ${ }^{*} P<0.05$ vs. quiescent, ${ }^{\#} P<0.05$ vs PDGF-BB + control lg.

Blocking integrin signaling with RGDS-blocking peptide. For blocking experiments, SMCs were pretreated with soluble RGDS integrin-blocking peptides (A9041, MilliporeSigma; $10 \mu \mathrm{M}, 30$ minutes) before addition of $\mathrm{cFn}$ or $\mathrm{pFn}$.
Immunocytochemistry. Immunostaining was performed in sections from stented human coronary arteries, mouse carotid arteries, and isolated mouse and human VSMCs. Stented human coronary artery sections were deplasticized, rehydrated, and pretreated with 
EDTA solution. Slides were incubated with Fn-EDA antibody (1:400; MilliporeSigma) or aSMA antibody (1:200; DAKO) overnight, and developed by the labeled streptavidin-biotin (LSAB) and NovaRed kit (Vector Laboratories). The images were captured by Axio Scan Z1 (Zeiss) using a $\times 20$ objective. Mouse arterial sections or cells were fixed in $4 \%$ paraformaldehyde in PBS for 10 minutes and permeabilized with $0.1 \%$ Triton X-100 (in PBS). After blocking with 5\% BSA for 1 hour, samples were incubated with antibodies specific for Fn-EDA+ (FN-3E2, 1:100; MilliporeSigma), vimentin (1:100; Abcam), osteopontin (1:100; Abcam), smooth muscle myosin heavy chain 11 (SM-MHC; 1:100; Abcam), smooth muscle 22-alpha (SM22 $\alpha ; 1: 100 ;$ Abcam), and $\alpha$-smooth muscle actin ( $\alpha$ SMA; 1:200; MilliporeSigma) overnight at $4^{\circ} \mathrm{C}$. The samples were washed and labeled with Alexa Fluor $488^{-}$ conjugated or Alexa Fluor 568-conjugated (1:400; Abcam) appropriate secondary antibodies for 1 hour at room temperature. Hoechst (5 $\mu \mathrm{g} / \mathrm{mL}$ ) staining was performed before mounting, and images were acquired with the same exposure time for all sections from different groups. A Nikon Eclipse Ti-U inverted fluorescent microscope equipped with a $\times 40 / 0.75$ and $\times 20 / 0.8$ Plan Apo lens cooled CCD camera and Nis Elements imaging software (Nikon) was used for imaging of VSMCs. For arterial sections, an Olympus fluorescent microscope (BX51) was used. Mean fluorescence intensity was quantified using Image J software as previously described (54). Protein colocalization was analyzed using Pearson's colocalization coefficients (JACoP; http://rsb.info.nih.gov/ij/plugins/track/jacop.html). Colocalization was considered positive for values ranging from 0.5 to 1 (55). Measurements were obtained from 2 different fields per murine section, and 4-6 different fields for human samples and cultured SMCs.

BrdU incorporation assay. Human and mouse aortic VSMCs were seeded into 96-well plates at a density of 3000 cells per well and then cultured in respective growth medium for 24 hours at $37^{\circ} \mathrm{C}$. Cells were then incubated with serum-free media for 48 hours. Cells were then washed and stimulated with PDGF $(20 \mathrm{ng} / \mathrm{mL}), \mathrm{cFn}(0-50 \mu \mathrm{g} / \mathrm{mL})$, or $\mathrm{pFn}(0-50 \mu \mathrm{g} / \mathrm{mL})$ for 24 hours. Cells were incubated with BrdU at a final concentration of $10 \mu \mathrm{M}$ for the last 12 hours of treatment (56). To assess the role of Akt or ERK in cFn-induced SMC proliferation, mouse aortic SMCs were treated with LY294002 $(10 \mu \mathrm{M})$ or U0126 $(10 \mu \mathrm{M})$ for 30 minutes before addition of $\mathrm{cFn}$ or $\mathrm{pFn}$. Carotid artery sections form BrdU-treated mice or cultured SMCs were fixed with $4 \%$ paraformaldehyde in PBS containing 0.1\% Triton X-100. DNA hydrolysis was then performed by treatment of the cells/sections with $2 \mathrm{M} \mathrm{HCl}$ for 20 minutes. Samples were incubated with a mixture of rabbit polyclonal anti- $\alpha$ SMA antibody (1:100; Abcam) and rat monoclonal antiBrdU antibody (1:200; Abcam) for 3 hours at $37^{\circ} \mathrm{C}$ and labeled with appropriate Alexa Fluor 488 and Alexa Fluor 568 secondary antibody (1:400; Abcam). Nuclei were stained with Hoechst $(5 \mu \mathrm{g} / \mathrm{mL})$ before mounting. The sections were examined using the Nikon Eclipse Ti-U fluorescent microscope, and the percentage of BrdU-positive cells over a total number of cells was determined using ImageJ software. In blocking experiments, human aortic SMCs were preincubated with Fn-EDA antibody (Fn-3E2, $10 \mu \mathrm{g} / \mathrm{mL}, 1$ hour) or an equal amount of isotype-matched control Ig before the addition of PDGF (39).

Cell cycle progression analysis. Serum-starved mouse aortic SMCs were stimulated with PDGF $(20 \mathrm{ng} / \mathrm{mL}), \mathrm{cFn}(20 \mu \mathrm{g} / \mathrm{mL})$, or pFn $(20 \mu \mathrm{g} / \mathrm{mL})$ for 24 hours. Cells were detached with trypsin and centrifuged at $150 \mathrm{~g}$ for 5 minutes, and the pellet was resuspended in hypotonic propidium iodide solution $(50 \mu \mathrm{g} / \mathrm{mL}$ with $0.03 \% \mathrm{NP}-40$ in $0.1 \%$ sodium citrate) for 20 minutes at $4^{\circ} \mathrm{C}$ (31). The DNA content in these cells was measured using a flow cytometer (LSR II; Becton Dickinson) and the FACSDiVa program, and then the cell-phase distribution was analyzed by Modfit software.

SMC migration. Cultured human and mouse aortic SMCs were seeded at $90 \%-95 \%$ confluence in 6-well culture plates and were allowed to adhere overnight. After serum starvation for 48 hours, a scratch was applied to the cell monolayer using a sterile $200-\mu \mathrm{L}$ pipette tip, and initial photomicrographs were taken using a Nikon Eclipse Ti-U phase-contrast microscope. Cells were then stimulated with PDGF or cFn $(20 \mu \mathrm{g} / \mathrm{mL})$ or $\mathrm{pFn}(20 \mu \mathrm{g} / \mathrm{mL})$, and the second set of images was obtained after 12 or 24 hours to measure SMC migration over the scratched area. The cell-free area for each well was measured using ImageJ software and the MRI wound healing tool plugin (57). Percentage of wound closure was calculated as the difference between the denuded area at 0 hours and the denuded area at 12 or 24 hours.

ELISA assay for TNF- $\alpha$ and IL-1 $\beta$. Serum-starved SMCs were treated for 24 hours with PDGF, cFn, or pFn as indicated above. The supernatant was collected, and cytokine levels were determined using commercially available mouse ELISA kits (R\&D Systems) according to the manufacturer's instructions.

Protein extraction and immunoblotting. Cellular and mouse tissue proteins were extracted using radioimmunoprecipitation assay (RIPA) lysis buffer containing protease inhibitor cocktail. The lysates were spun down at $4^{\circ} \mathrm{C}(16,000 \mathrm{~g})$ for 15 minutes. Protein quantification in the supernatant was done using bicinchoninic acid reagent. Equal amounts of total protein samples were resolved by SDS-PAGE, transferred onto a PVDF membrane, and blocked with TBST containing 5\% BSA for 1 hour. The membrane was then incubated with primary antibodies against phospho-Akt1 (S473), total Akt (pan), phospho-Akt2 (Ser474), total Akt2 (D6G4), phospho-mTOR (Ser2448), total mTOR (9102), phospho-NF-кB p65 (Ser536), total NF-кB p65 (D14E12), phospho-FAK (Tyr397), FAK, P-Src family (Tyr416), Src, phospho-p44/42 MAPK (Erk1/2) (Thr202/Tyr204), p44/42 MAPK (Erk1/2), Fn, and TLR4. All antibodies were diluted $1: 1000$ in TBST containing $5 \%$ $\mathrm{BSA}$, and the proteins were normalized with $\beta$-actin (MilliporeSigma) and respective total protein content. The specific bands were detected by SuperSignal West Femto Maximum Sensitivity substrate (Thermo Fisher Scientific) using ChemiDoc Imaging System (Bio-Rad). Densitometric analysis of the gels was done using ImageJ software. See complete unedited blots in the supplemental material.

Detection of apoptotic cells by TUNEL staining. Apoptotic cells within the carotid artery sections were detected using the In Situ Cell Death Detection Kit, Fluorescein (11684809910, Roche). Aortic sections were permeabilized with $0.2 \%$ Triton and subsequently incubated with TUNEL reaction mixture for 60 minutes at $37^{\circ} \mathrm{C}$ in a humidified chamber. Upon staining with Hoechst $(5 \mu \mathrm{g} / \mathrm{mL})$, the sections were examined under a fluorescence microscope (Olympus BX51). Two fields were selected to cover almost the entire tissue section. Percentage of apoptotic cells over a total number of cells was quantified using ImageJ software as described previously (58).

Bone marrow transplantation. We performed bone marrow transplantation (BMT) in Fn-EDA ${ }^{\mathrm{f} / \mathrm{fl}} \mathrm{Tie}^{\mathrm{C}} \mathrm{Cre}^{+} \mathrm{Apoe}^{-/-}$with bone marrow from $\mathrm{Fn}-\mathrm{EDA}^{\mathrm{f} / \mathrm{fl}} \mathrm{Apoe}^{-/-}$mice because of the evidence that Tie2 is expressed by hematopoietic cells in addition to endothelial cells. This strategy resulted in a mutant mouse that is deficient for Fn-EDA only in endothelial cells (Fn-EDA ${ }^{\mathrm{fl} / \mathrm{ll}} \mathrm{Tie}^{\mathrm{C}} \mathrm{Cre}^{+} \mathrm{Apoe}^{-/-}$mouse). Recipient mice at 7-8 weeks of age were irradiated with 2 doses of $6.5 \mathrm{~Gy}$ at an interval 
of 4 hours between the first and second irradiations. Bone marrow cells were aseptically extracted from excised femurs and tibiae of euthanized Fn-EDA ${ }^{\mathrm{fl} / \mathrm{ll}} \mathrm{Apoe}^{-/-}$mice. Bone marrow cells $\left(1 \times 10^{7}\right)$ were resuspended in sterile PBS and injected into the retro-orbital plexus of lethally irradiated recipient mice. After transplantation, mice were maintained in sterile cages and fed autoclaved food and water ad libitum. BMT success was analyzed after 4 weeks by PCR to check the presence of the genomic DNA (of the respective donor mice) in peripheral blood mononuclear cells from transplanted mice. Complete blood counts were obtained using an automated veterinary hematology analyzer (ADVIA, Siemens Healthineers; Supplemental Table 3) to ascertain that BMT did not affect the number of bone marrow-derived blood cells.

Statistics. Results are reported as the mean \pm SEM. The statistical significance of the difference between means was assessed using the unpaired 2-tailed Student's $t$ test (for the comparison of 2 groups) or by ANOVA followed by Bonferroni's multiple-comparisons tests (parametric data of more than 2 groups). $P$ less than 0.05 was considered significant. The number of animals $(n=10-12)$ in each group was based on power calculations for the primary parameter (neointimal area) with a power of 0.8 , an SD of $10 \%$, and $\alpha=0.05, \beta=0.2$. GraphPad Prism (version 7.0) was used for statistical analysis. The Shapiro-Wilk test was used to check normality, and Bartlett's test was used to check equal variance.

Study approval. Studies involving the use of human stented and diseased autopsy specimens were approved by the Institutional Review Board of the CVPath Institute and the University of Iowa, respectively. The University of Iowa Animal Care and Use Committee approved all procedures, and studies were performed according to the current Animal Research: Reporting of In Vivo Experiments guidelines (https://www.nc3rs.org.uk/arrive-guidelines).

\section{Author contributions}

MJ designed the study, performed experiments, analyzed and interpreted results, and cowrote the manuscript; ND generated and validated endothelial cell- and smooth muscle cell-specific $\mathrm{Fn}_{-} \mathrm{EDA}^{-/-}$mice and performed experiments; PD and MRC performed experiments and analyzed results; MKN helped with flow cytometry experiments; and AC, LG, and AVF from the CVPath Institute performed immunostaining in stented human coronary artery sections. SRL was conceptually involved and provided critical input for revising the manuscript; and AKC directed the project, designed the study, interpreted results, and cowrote the manuscript.

\section{Acknowledgments}

Andres F. Muro provided EDA-containing and EDA-lacking recombinant peptides. The AKC laboratory is supported by grants from the NIH (R35HL139926 and R01NS109910) and by Established Investigator Award 18EIA33900009 from the American Heart Association. The AVF laboratory is supported by grants from the CVPath Institute; from the Leducq Foundation Transatlantic Networks of Excellence (grant 18CVD02) to the PlaqOmics Research Network (AVF); and from the Research Rotation Program of the Medical Faculty of RWTH Aachen University (to AC).

Address correspondence to: Anil K. Chauhan, University of Iowa, Department of Internal Medicine, 3120 Medical Labs, Iowa City, Iowa 52242, USA. Phone: 319.335.6525; Email: anil-chauhan@uiowa.edu.
1. Indolfi C, Mongiardo A, Curcio A, Torella D. Molecular mechanisms of in-stent restenosis and approach to therapy with eluting stents. Trends Cardiovasc Med. 2003;13(4):142-148.

2. Raines EW. The extracellular matrix can regulate vascular cell migration, proliferation, and survival: relationships to vascular disease. Int J Exp Pathol. 2000;81(3):173-182.

3. Thyberg J, Blomgren K, Roy J, Tran PK, Hedin U. Phenotypic modulation of smooth muscle cells after arterial injury is associated with changes in the distribution of laminin and fibronectin. J Histochem Cytochem. 1997;45(6):837-846.

4. Hedin U, Bottger BA, Forsberg E, Johansson S, Thyberg J. Diverse effects of fibronectin and laminin on phenotypic properties of cultured arterial smooth muscle cells. J Cell Biol. 1988;107(1):307-319.

5. White ES, Baralle FE, Muro AF. New insights into form and function of fibronectin splice variants. J Pathol. 2008;216(1):1-14.

6. Wang Y, et al. Plasma fibronectin supports hemostasis and regulates thrombosis. J Clin Invest. 2014;124(10):4281-4293.

7. Ffrench-Constant C. Alternative splicing of fibronectin--many different proteins but few different functions. Exp Cell Res. 1995;221(2):261-271.

8. Ffrench-Constant C, Van de Water L, Dvorak HF, Hynes RO. Reappearance of an embryonic pattern of fibronectin splicing during wound healing in the adult rat. J Cell Biol. 1989;109(2):903-914.
9. Muro AF, et al. Regulated splicing of the fibronectin EDA exon is essential for proper skin wound healing and normal lifespan.J Cell Biol. 2003;162(1):149-160.

10. Glukhova MA, et al. Expression of extra domain A fibronectin sequence in vascular smooth muscle cells is phenotype dependent. J Cell Biol. 1989;109(1):357-366.

11. Muro AF, et al. An essential role for fibronectin extra type III domain A in pulmonary fibrosis. Am J Respir Crit Care Med. 2008;177(6):638-645.

12. Bhattacharyya S, et al. FibronectinEDA promotes chronic cutaneous fibrosis through Toll-like receptor signaling. Sci Transl Med. 2014;6(232):232ra50.

13. Okamura Y, et al. The extra domain A of fibronectin activates Toll-like receptor 4.J Biol Chem. 2001;276(13):10229-10233.

14. Lee GL, et al. TLR4-activated MAPK-IL-6 axis regulates vascular smooth muscle cell function. Int JMol Sci. 2016;17(9):E1394.

15. Morla AO, Mogford JE. Control of smooth muscle cell proliferation and phenotype by integrin signaling through focal adhesion kinase. Biochem Biophys Res Commun. 2000;272(1):298-302.

16. Dhanesha N, Ahmad A, Prakash P, Doddapattar P, Lentz SR, Chauhan AK. Genetic ablation of extra domain A of fibronectin in hypercholesterolemic mice improves stroke outcome by reducing thrombo-inflammation. Circulation. 2015;132(23):2237-2247.
17. Doddapattar P, et al. Fibronectin splicing variants containing extra domain A promote atherosclerosis in mice through Toll-like receptor 4. Arterioscler Thromb Vasc Biol. 2015;35(11):2391-2400.

18. Doddapattar P, Jain M, Dhanesha N, Lentz SR, Chauhan AK. Fibronectin containing extra domain A induces plaque destabilization in the innominate artery of aged apolipoprotein E-deficient mice. Arterioscler Thromb Vasc Biol. 2018;38(3):500-508.

19. Tan MH, Sun Z, Opitz SL, Schmidt TE, Peters JH, George EL. Deletion of the alternatively spliced fibronectin EIIIA domain in mice reduces atherosclerosis. Blood. 2004;104(1):11-18.

20. Pulakazhi Venu VK, et al. Fibronectin extra domain A stabilises atherosclerotic plaques in apolipoprotein E and in LDL-receptor-deficient mice. Thromb Haemost. 2015;114(1):186-197.

21. Paudel B, Xuan GJ, Chun ZF. Analysis of clinical factors affecting the restenosis following percutaneous coronary intervention. Nepal Med Coll J. 2005;7(2):101-106.

22. Wildgruber $\mathrm{M}$, et al. Increased intermediate $\mathrm{CD} 14^{++} \mathrm{CD} 16^{++}$monocyte subset levels associate with restenosis after peripheral percutaneous transluminal angioplasty. Atherosclerosis. 2016;253:128-134.

23. Chaabane C, Otsuka F, Virmani R, BochatonPiallat ML. Biological responses in stented arteries. Cardiovasc Res. 2013;99(2):353-363.

24. Meng R, Mi X, Sun D. Risk factors for recurrent 
carotid-artery stenosis following stenting treatment. Med Sci Monit. 2019;25:2429-2434.

25. Chauhan AK, Iaconcig A, Baralle FE, Muro AF. Alternative splicing of fibronectin: a mouse model demonstrates the identity of in vitro and in vivo systems and the processing autonomy of regulated exons in adult mice. Gene. 2004;324:55-63.

26. Hedin U, Thyberg J. Plasma fibronectin promotes modulation of arterial smooth-muscle cells from contractile to synthetic phenotype. Differentiation. 1987;33(3):239-246.

27. Han M, Wen JK, Zheng B, Cheng Y, Zhang C. Serum deprivation results in redifferentiation of human umbilical vascular smooth muscle cells. Am J Physiol Cell Physiol. 2006;291(1):C50-C58.

28. Kato M, Kyogoku M. Competence growth factors evoke the phenotypic transition of arterial smooth muscle cells. Ann N Y Acad Sci. 1990;598:232-237.

29. Fernández-Hernando C, József L, Jenkins D, Di Lorenzo A, Sessa WC. Absence of Akt1 reduces vascular smooth muscle cell migration and survival and induces features of plaque vulnerability and cardiac dysfunction during atherosclerosis. Arterioscler Thromb Vasc Biol. 2009;29(12):2033-2040.

30. Jin Y, et al. Opposing actions of AKT (protein kinase B) isoforms in vascular smooth muscle injury and therapeutic response. Arterioscler Thromb Vasc Biol. 2017;37(12):2311-2321.

31. Jain M, Singh A, Singh V, Barthwal MK. Involvement of interleukin-1 receptor-associated kinase-1 in vascular smooth muscle cell proliferation and neointimal formation after rat carotid injury. Arterioscler Thromb Vasc Biol. 2015;35(6):1445-1455.

32. Kelsh R, You R, Horzempa C, Zheng M, McKeown-Longo PJ. Regulation of the innate immune response by fibronectin: synergism between the III-1 and EDA domains. PLoS One. 2014;9(7):e102974.

33. Barnes JL, Hastings RR, De la Garza MA. Sequential expression of cellular fibronectin by platelets, macrophages, and mesangial cells in proliferative glomerulonephritis. Am J Pathol. 1994;145(3):585-597.

34. van Keulen JK, et al. Levels of extra domain A containing fibronectin in human atherosclerotic plaques are associated with a stable plaque phenotype. Atherosclerosis. 2007;195(1):e83-e91.
35. Lemańska-Perek A, Krzyżanowska-Gołąb D, Pupek M, Klimeczek P, Witkiewicz W, Kątnik-Prastowska I. Analysis of soluble molecular fibronectin-fibrin complexes and EDA-fibronectin concentration in plasma of patients with atherosclerosis. Inflammation. 2016;39(3):1059-1068.

36. Peters JH, Maunder RJ, Woolf AD, Cochrane CG, Ginsberg MH. Elevated plasma levels of ED1+ (“cellular") fibronectin in patients with vascular injury. J Lab Clin Med. 1989;113(5):586-597.

37. Shekhonin BV, Domogatsky SP, Idelson GL, Koteliansky VE, Rukosuev VS. Relative distribution of fibronectin and type I, III, IV, V collagens in normal and atherosclerotic intima of human arteries. Atherosclerosis. 1987;67(1):9-16.

38. Losino $\mathrm{N}$, et al. EDA-containing fibronectin increases proliferation of embryonic stem cells. PLoS One. 2013;8(11):e80681.

39. Kohan M, Muro AF, White ES, Berkman N. EDA-containing cellular fibronectin induces fibroblast differentiation through binding to alpha4beta7 integrin receptor and MAPK/Erk 1/2-dependent signaling. FASEB J. 2010;24(11):4503-4512.

40. Prakash P, Kulkarni PP, Lentz SR, Chauhan AK. Cellular fibronectin containing extra domain A promotes arterial thrombosis in mice through platelet Toll-like receptor 4 . Blood. 2015;125(20):3164-3172.

41. Zhang LL, et al. PPAR $\gamma$ attenuates intimal hyperplasia by inhibiting TLR4-mediated inflammation in vascular smooth muscle cells. Cardiovasc Res. 2011;92(3):484-493.

42. Hauck CR, Hsia DA, Schlaepfer DD. Focal adhesion kinase facilitates platelet-derived growth factor-BB-stimulated ERK2 activation required for chemotaxis migration of vascular smooth muscle cells. J Biol Chem. 2000;275(52):41092-41099.

43. Yin Q, et al. LPS Promotes vascular smooth muscle cells proliferation through the TLR4/Rac1/ Akt signalling pathway. Cell Physiol Biochem. 2017;44(6):2189-2200.

44. Jiang D, et al. Positive feedback regulation of proliferation in vascular smooth muscle cells stimulated by lipopolysaccharide is mediated through the TLR 4/Rac1/Akt pathway. PLoS One. 2014;9(3):e92398.

45. Manning BD, Toker A. AKT/PKB signaling: navigating the network. Cell. 2017;169(3):381-405.

46. Hegner B, et al. mTOR regulates vascular smooth muscle cell differentiation from human bone marrow-derived mesenchymal progenitors. Arterioscler Thromb Vasc Biol. 2009;29(2):232-238.

47. Peters JH, Sporn LA, Ginsberg MH, Wagner DD. Human endothelial cells synthesize, process, and secrete fibronectin molecules bearing an alternatively spliced type III homology (ED1). Blood. 1990;75(9):1801-1808.

48. Dhanesha N, et al. Fn-EDA (fibronectin containing extra domain A) in the plasma, but not endothelial cells, exacerbates stroke outcome by promoting thrombo-inflammation. Stroke. 2019;50(5):1201-1209.

49. Muro AF, et al. Regulated splicing of the fibronectin EDA exon is essential for proper skin wound healing and normal lifespan. JCell Biol. 2003;162(1):149-160.

50. Cai J, et al. HMGB1-driven inflammation and intimal hyperplasia after arterial injury involves cell-specific actions mediated by TLR4. Arterioscler Thromb Vasc Biol. 2015;35(12):2579-2593.

51. Li W, et al. The multifunctional $\mathrm{Ca} 2+/$ calmodulin-dependent kinase II delta (CaMKIIdelta) controls neointima formation after carotid ligation and vascular smooth muscle cell proliferation through cell cycle regulation by $\mathrm{p} 21$. J Biol Chem. 2011;286(10):7990-7999.

52. Khanna V, et al. Cholesterol diet withdrawal leads to an initial plaque instability and subsequent regression of accelerated iliac artery atherosclerosis in rabbits. PLoS One. 2013;8(10):e77037.

53. RayJL, Leach R, Herbert JM, Benson M. Isolation of vascular smooth muscle cells from a single murine aorta. Methods Cell Sci. 2001;23(4):185-188.

54. Jensen EC. Quantitative analysis of histological staining and fluorescence using Image J. Anat Rec (Hoboken). 2013;296(3):378-381.

55. Nisticò R, et al. Presynaptic c-Jun N-terminal kinase 2 regulates NMDA receptor-dependent glutamate release. Sci Rep. 2015;5:9035.

56. Uemura Y, et al. Adipose-derived factor CTRP9 attenuates vascular smooth muscle cell proliferation and neointimal formation. FASEB J. 2013;27(1):25-33.

57. Cormier N, Yeo A, Fiorentino E, Paxson J. Optimization of the wound scratch assay to detect changes in murine mesenchymal stromal cell migration after damage by soluble cigarette smoke extract. J Vis Exp. 2015;(106):e53414.

58. Helmy IM, Azim AM. Efficacy of ImageJ in the assessment of apoptosis. Diagn Pathol. 2012;7:15. 